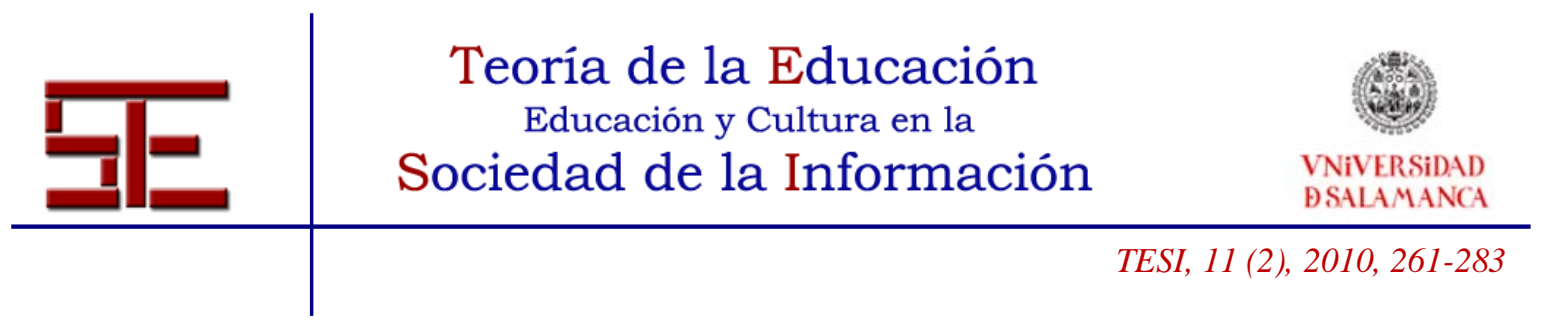

\title{
UTILIZACIÓN DE SOFTWARE DE CORRECCIÓN AUTOMÁTICA EN EL CAMPO DE LAS CIENCIAS DE LA SALUD
}

\section{Resumen:}

Estamos viviendo una época de cambios profundos en la educación universitaria. La implantación del plan de Bolonia nos ha llevado a plantear nuevas metodologías docentes, a revisar el papel del estudiante, la evaluación por competencias, la incorporación de las TIC. Hechos impensables hace poco más de una década.

Entre las diferentes plataformas informáticas, cabe destacar las que permiten corrección automática de ejercicios, porque son instrumentos de un gran interés pedagógico ya que evalúan al instante al alumnado y aportan un feedback del conocimiento que tiene en forma de mensaje de ayuda o de nota. Si la potencia de estas herramientas la sumamos a la de Internet, usando un entorno de e-learning, el resultado permitirá trabajar, corregir, evaluar, resolver dudas, etc., desde cualquier lugar y a cualquier hora.

Este trabajo presenta parte de una plataforma y los resultados de su utilización en el ámbito de las ciencias de la salud.

Palabras clave: Herramientas corrección automática, e-learning, aprendizaje autónomo

Ferrán Prados Carrasco, Gloria Mateu Figueras, Carmen Echazarreta Soler, Jordi Poch García, Josep Soler Masó e Imma Boada Oliveras 


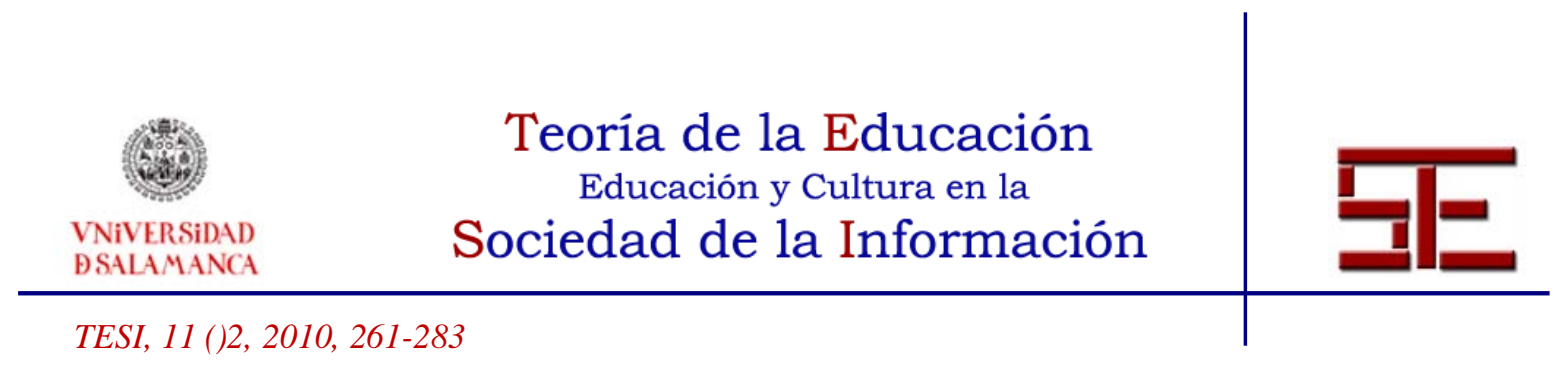

\title{
USING AUTOMATIC CORRECTION SOFTWARE IN THE FIELD OF HEALTH SCIENCES
}

\begin{abstract}
:
We live in an era of profound changes in university education. The implementation of Bologna plan has led us to raise new teaching methodologies, to review the role of the student, competency assessment, the incorporation of ICT. Unthinkable acts, one or two decade ago.

The TIC concept is very broad and is attributed to the media, processes and content usage. Inside the supports and platforms, we stress tools that allow automatic correction of exercises, because they are instruments of great educational value because instantly they assess students and provide instant feedback about the knowledge that they have either as message support or note. If the power of these tools, we add the Internet, using e-learning environment, the results allow us to work, edit, evaluate, resolve doubts, and so on, anywhere, anytime.
\end{abstract}

We present part of a platform and the results of its use in the field of health sciences.

Keywords: Automatic correction tools, e-learning, self-learning

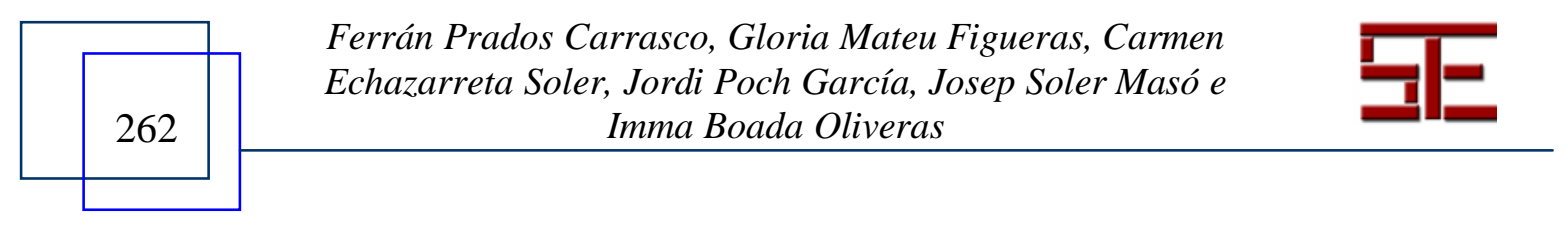




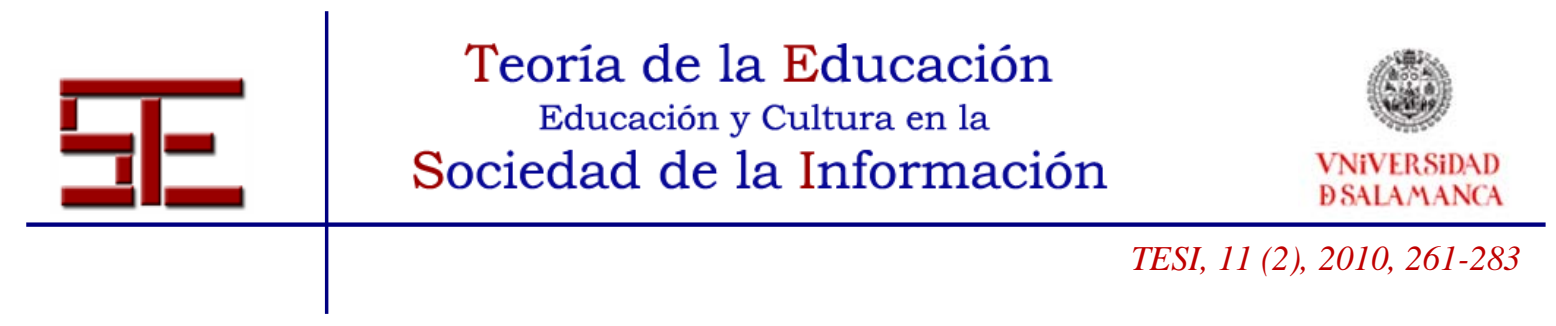

\section{UTILIZACIÓN DE SOFTWARE DE CORRECCIÓN AUTOMÁTICA EN EL CAMPO DE LAS CIENCIAS DE LA SALUD}

Fecha de recepción: 19/11/2009; fecha de aceptación: 02/04/2010; fecha de publicación: 05/07/2010

Ferrán Prados Carrasco

ferran.prados@udg.edu

Universidad de Girona

Gloria Mateu Figueras

gloria.mateu@udg.edu

Universidad de Girona

Jordi Poch García

jordi.poch@udg.edu

Universidad de Girona

Josep Soler Masó

josep.soler@udg.edu

Universidad de Girona

Imma Boada Oliveras

imma.boada@udg.edu

Universidad de Girona

Carmen Echazarreta Soler

carmen.echazarreta@udg.edu

Universidad de Girona

Ferrán Prados Carrasco, Gloria Mateu Figueras, Carmen

Echazarreta Soler, Jordi Poch García, Josep Soler Masó e Imma Boada Oliveras 


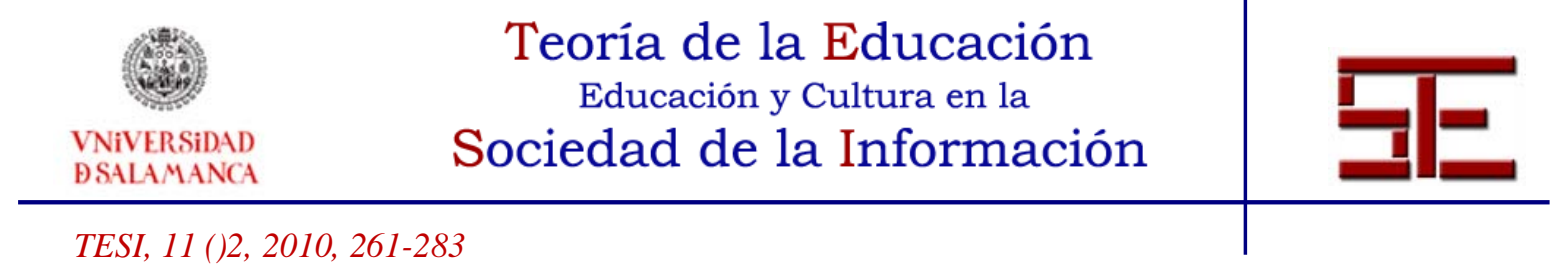

\section{1.- INTRODUCCIÓN}

Estamos viviendo una época de cambios profundos en la educación universitaria. La implantación del plan de Bolonia ha comportado el despliegue de los créditos ECTS (European Credit Transfer and Accumulation System). Este nuevo concepto de crédito académico supone desarrollar nuevas metodologías docentes que sitúen al estudiante en el centro de su aprendizaje y evalúen competencias. En este contexto, las TIC (Tecnologías de la Información y la Comunicación) son indudablemente instrumentos que permiten el diseño de una metodología de innovación docente. En definitiva, todo esto ha provocado una revolución en las clases universitarias que vivían, hasta hace poco más de una década, afincadas en un modelo educativo tradicional, fundamentado en las clases magistrales.

Está claro que en esta revolución hay dos actores muy importantes que conviven en plena simbiosis: el plan de Bolonia y las TIC. Las herramientas TIC han encontrado en el plan de Bolonia el cambio metodológico y de planeamiento del aprendizaje necesario para su implantación y utilización (Echazarreta, 2009). Por su parte, el plan de Bolonia ha encontrado en las TIC su aliado perfecto para llevar a cabo la idea de situar el alumno en el centro de su aprendizaje (Esteve, 2009). Esta relación simbiótica ha supuesto la utilización de multitud de herramientas TIC en la docencia universitaria. Experiencias como el uso de:

- Simuladores (Davidovitch, 2006; Lammers, 2008)

- Tutoriales interactivos (Spence, 1994; Buzzell, 2002)

- Herramientas de web 2.0: wiki, blog, twitter, etc. (Bruns, 2005; Prados, 2009)

- Portafolios (Prados, 2008; Barberà, 2009)

- Redes sociales: Facebook, SecondLife, Orkut, etc.

La utilización de metodologías activas en contraposición de las metodologías docentes tradicionales ha de suponer que el alumno aprenda haciendo (Benito, 2005). Las TIC se han convertido en una pieza más del diseño curricular, son una herramienta ideal para seguir el trabajo del alumno tanto dentro de clase como fuera. Pero podemos diferenciar cuatro tipos de herramientas TIC según el tipo de feedback que recibe el alumno al utilizarlas. Estas se podrían ordenar de menos interactiva a más del siguiente modo:

- Difusión de contenidos: pueden ser páginas web, simuladores, tutoriales, etc. que muestran al alumno una información y que le permiten hacer una navegación dirigida o anárquica por el conocimiento que se quiere transmitir.

- Grupales: en esta categoría encontraríamos los portafolios, wikis, glosarios, etc. herramientas que nos permiten desarrollar metodologías de trabajo en grupo

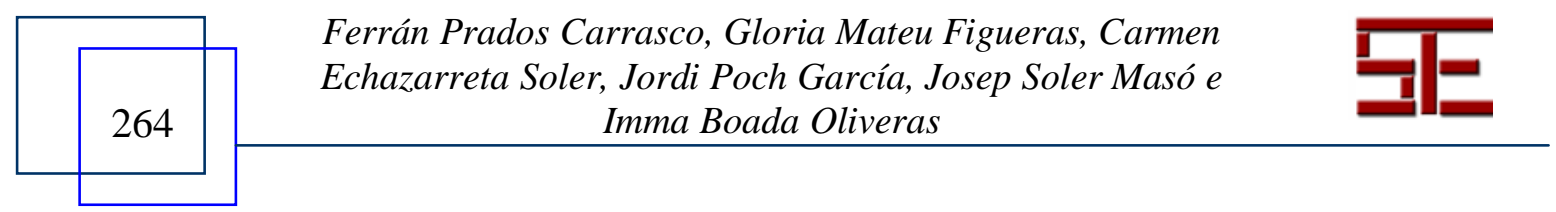




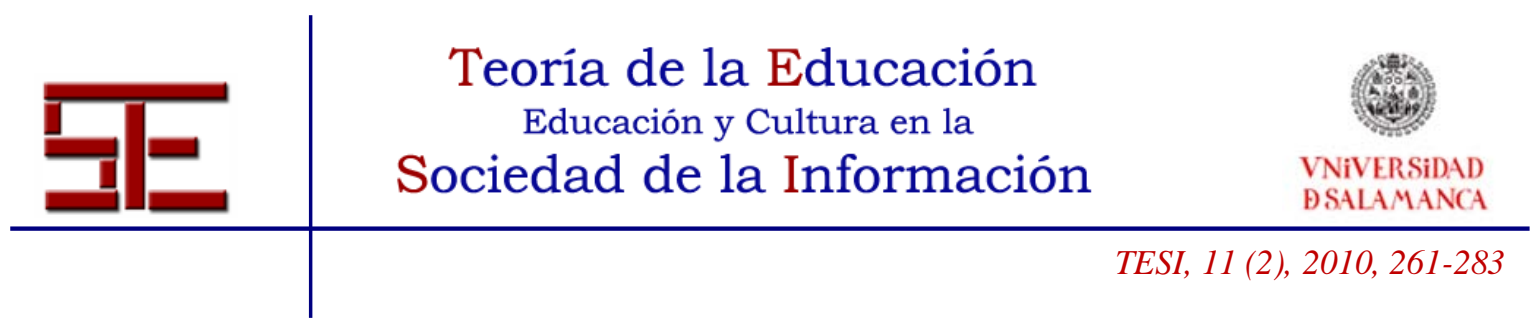

dónde el alumno hace diferente aportaciones y el feedback viene dado por las aportaciones del profesor o de sus compañeros.

- Autoevaluaciones: aquí podríamos situar herramientas de difusión de contenidos que se han ampliado con cuestionarios que le sirven al alumno para autoevaluar su aprendizaje. Así mismo, también encontraríamos la utilización de rúbricas como método autoevaluativo.

- Corrección automática: en este grupo encontraríamos el conjunto de herramientas que dan un feedback al instante a partir de una solución propuesta por un alumno. En este grupo podemos encontrar herramientas como los típicos cuestionarios tipo test, módulos de Moodle que permiten corrección automática, HotPotatoes, plataformas d'e-learning especializadas en la corrección automática como ACME.

Podemos asegurar que las TIC se han convertido en uno de los pilares básicos de la enseñanza universitaria. Esta afirmación viene corroborada por los datos reflejados en el estudio realizado para el Ministerio de Educación, Cultura y Ciencia (Paz, 2008) en el cual de las 73 universidades españolas, en el 2008, se contabilizaron 68 con campus virtuales, entendiendo como tal un espacio organizativo de la docencia ofrecida por la universidad a través de Internet.

Habitualmente la finalidad real de estas plataformas de e-learning es publicar material docente, o bien para mantener la comunicación con el alumnado, ya sea a través de email (tutorías virtuales) o a través de foros. Desde nuestro punto de vista, el uso de estas plataformas únicamente para publicar el material de las distintas asignaturas y como sistema de comunicación supone infrautilizar las posibilidades que puede ofrecer un entorno de e-learning.

Asimismo las herramientas que permiten la corrección automática son herramientas de un gran interés pedagógico ya que evalúan al instante al alumnado y aportan un feedback sobre el conocimiento que tiene ya sea en forma de mensaje de ayuda o de nota. Si la potencia de estas herramientas la sumamos a la de Internet, usando un entorno de e-learning, esto permitirá trabajar, corregir, evaluar, resolver dudas, etc., desde cualquier lugar y a cualquier hora.

Conscientes de las posibilidades que ofrece el e-learning nos propusimos avanzar un poco más, y nos pusimos a diseñar e implementar un entorno virtual capaz de corregir y evaluar automáticamente el trabajo realizado por el alumnado en cualquier asignatura (Prados 2004). Una herramienta con estas capacidades ofrece numerosas ventajas: nos permite realizar una evaluación continuada del alumnado, con la corrección automática le facilitaremos la corrección de sus ejercicios ofreciendo de esta forma el equivalente a

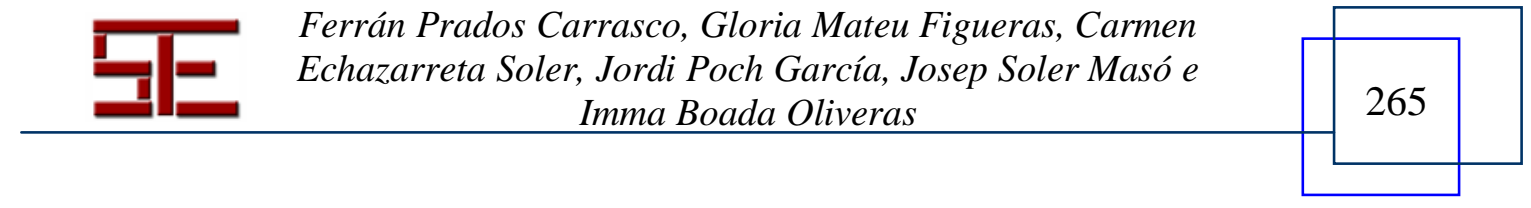




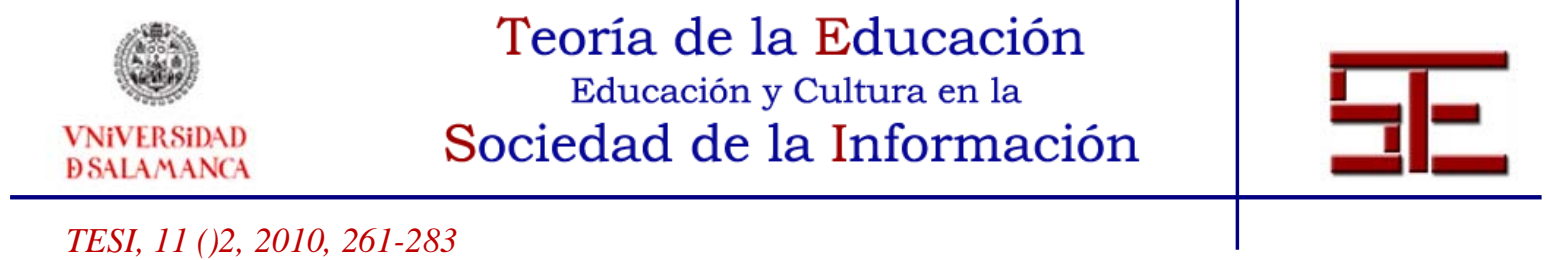

un profesor virtual 24 horas al día, así como la posibilidad de corregir los déficit de formación que pudiera tener en materias básicas.

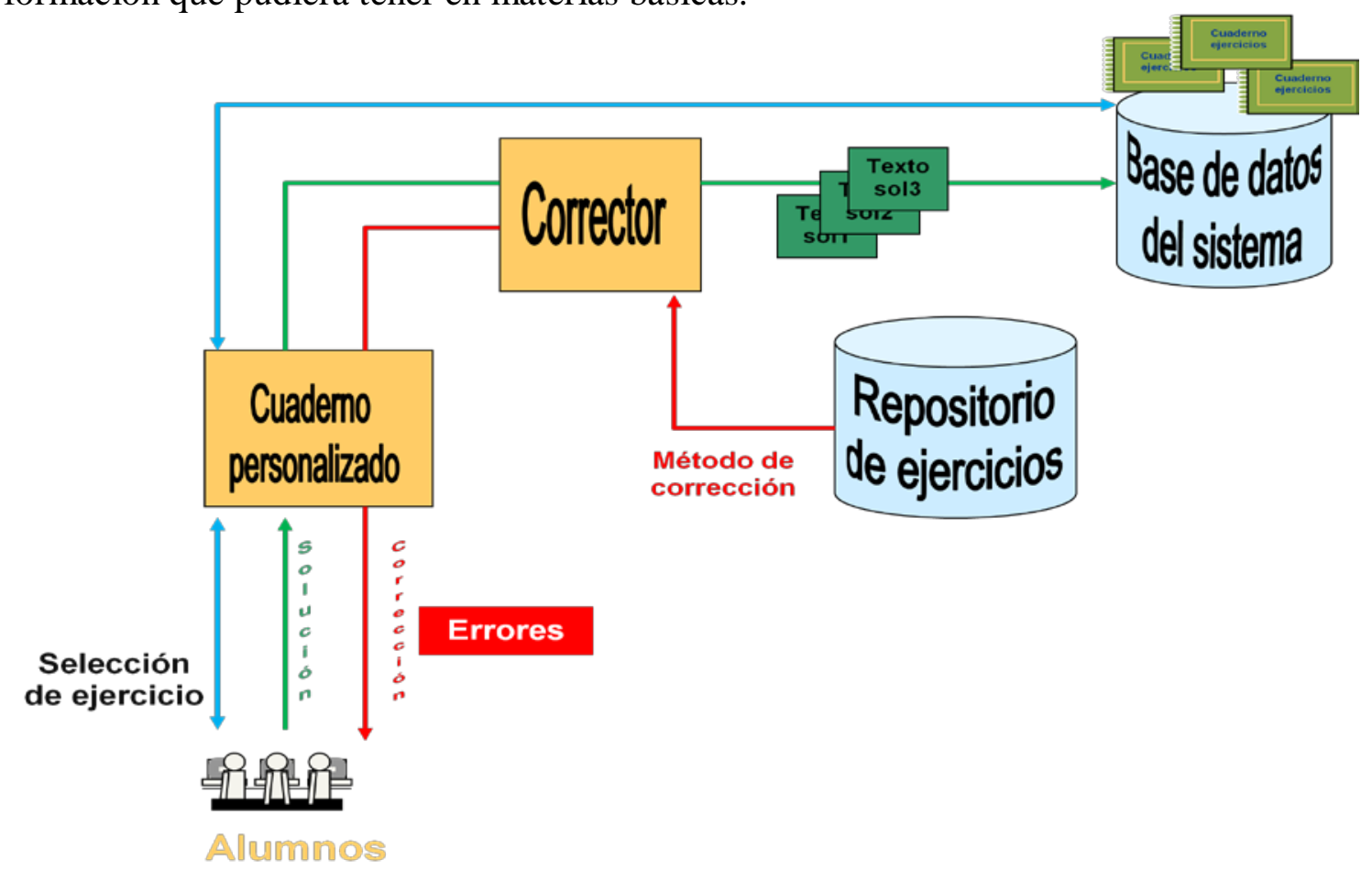

Figura 1. Esquema básico del funcionamiento de la plataforma ACME

El punto clave para alcanzar nuestro objetivo radicaba en el diseño y desarrollo de un módulo que permitiera la corrección automática de cualquier tipo de ejercicio (Figura 1). Dicho módulo fue desarrollado por un grupo de profesores del departamento de Informática y Matemática Aplicada de la Universidad de Girona (UdG), e integrado por otros módulos en una plataforma común a la que denominamos ACME (acrónimo de Evaluación Continuada y Mejora de la Enseñanza. ACME). Actualmente ACME se ha implantado en la Escuela Politécnica Superior, en la Escuela Universitaria de Enfermería, en las Facultades de Medicina, de Economía, de Turismo, Publicidad y Relaciones Públicas, de Ciencias y de Educación de la Universidad de Girona como plataforma virtual de soporte docente a las clases presenciales y con resultados muy satisfactorios.

\section{2.- HERRAMIENTAS DE CORRECCIÓN AUTOMÁTICA}

Los módulos de corrección automática se han ido incorporando a las plataformas de elearning en la última década. El ejemplo clásico son los correctores tipo test, que permiten al usuario seleccionar una de las respuestas. A partir de aquí podemos

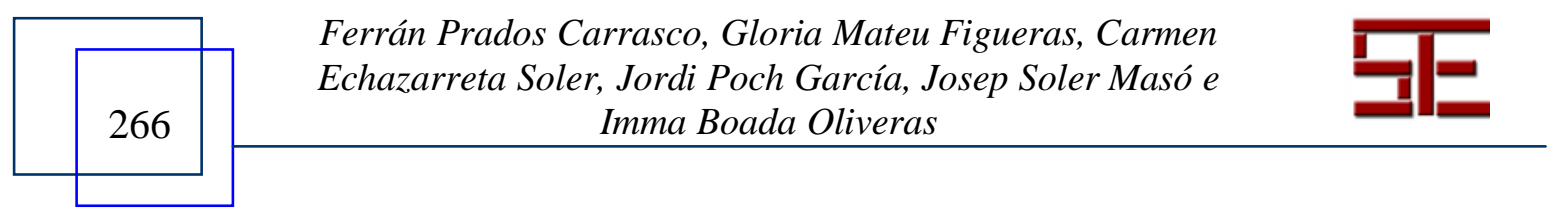




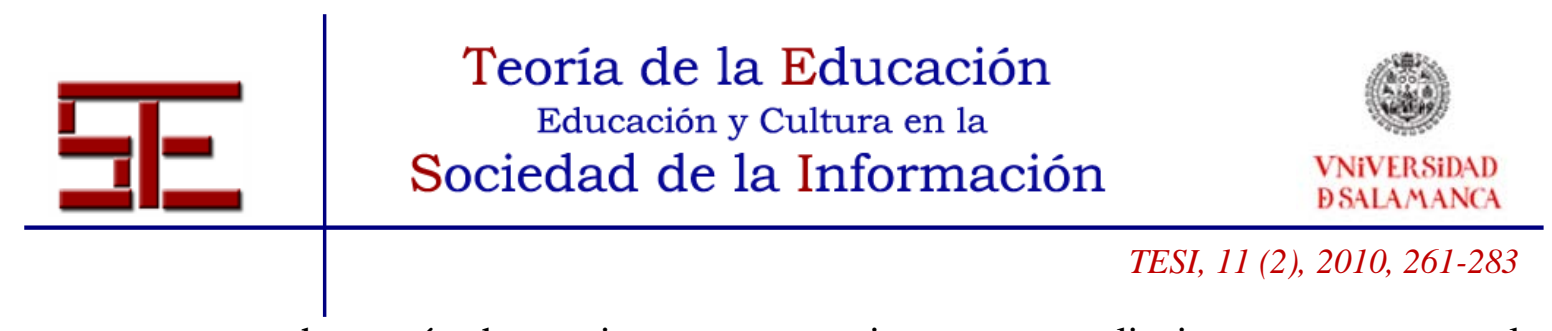

encontrar muchas más herramientas con variantes o ampliaciones, como es el HotPotatoes, plugins de plataformas de e-learning como el Moodle, WebCT, Sakai,...

Existen también iniciativas dónde se ha conectado software específico a páginas web con la finalidad de automatizar la corrección de ejercicios. Un ejemplo claro lo encontramos en plataformas web a través de las cuales podemos probar un programa informático (Boada 2004). Estas plataformas permiten enviar el código fuente del algoritmo y ellas mismas se encargan de compilar y ejecutar diferentes test sobre el algoritmo, con la finalidad de evaluar éste. También hallamos ejemplos conectando software de cálculo simbólico con interfícies web cómo la calculadora Wiris ${ }^{1}$. Usualmente estas herramientas destacan por ser específicas para la corrección de forma automática de algún tipo de ejercicio en concreto.

La plataforma ACME que presentamos en este trabajo se enmarca dentro de este grupo, si bien cabe destacar que engloba una amplia variedad de tipologías de ejercicios que se corrigen de forma automática. ACME se caracteriza por ser una herramienta modular que puede corregir automáticamente muchos tipos de ejercicios pertenecientes a distintas áreas de conocimiento como matemáticas (Soler 2002), informática (Boada 2004), base de datos (Soler 2009), electrónica, economía, mecánica, física, química, etc., y también, ejercicios del ámbito de las ciencias de la salud. Además, la plataforma ACME dispone de un módulo generador de enunciados a partir de un ejercicio base, y todos los enunciados generados son corregidos automáticamente (Prados 2005).

Las principales ventajas de combinar un generador de enunciados con un corrector automático son:

- Feedback instantáneo para el alumno, gracias a los mensajes que puede recibir del corrector.

- Facilita el seguimiento del grupo ya que la tarea de corregir se encarga la máquina, el profesor se puede centrar más en los casos que necesiten atención personalizada.

- Ejercicios personalizados para cada alumno, que a su vez disminuye la posibilidad de copia entre ellos.

- Funcionamiento ininterrumpido.

- Se puede aplicar con excelentes resultados en grupos grandes de alumnos (Soler 2006).

A continuación pasamos a describir el módulo de generación automática y algunas de las topologías de ejercicios con posibles usos en el ámbito de la salud.

Ferrán Prados Carrasco, Gloria Mateu Figueras, Carmen Echazarreta Soler, Jordi Poch García, Josep Soler Masó e Imma Boada Oliveras 


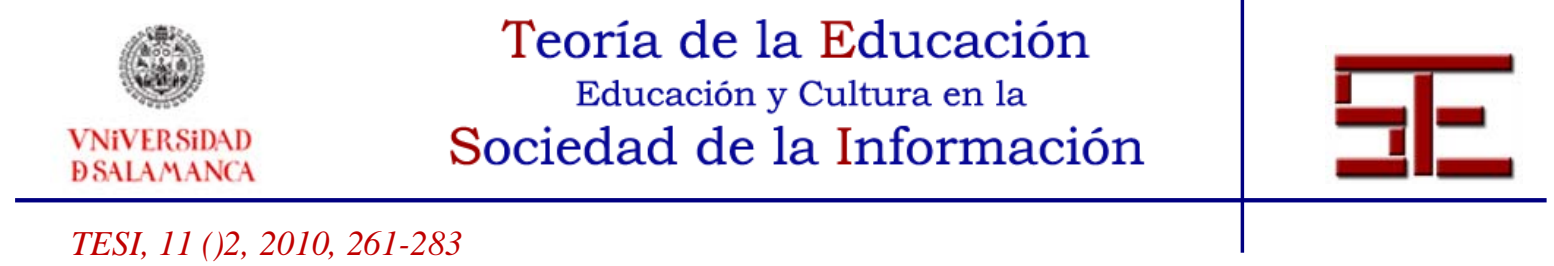

\section{1-Generadores de enunciados}

La corrección automática de ejercicios es un avance muy importante en el campo del elearning, pero si a este le añadimos un generador automático de ejercicios aún ampliamos todavía más su potencia y su utilidad. Un generador de ejercicios es un programa capaz de crear desenas, centenares o miles de enunciados a partir de un enunciado base. Estos enunciados base pueden disponer de una o varias redacciones que el generador escoge al azar, y cada una de estas redacciones a su vez dispone de parámetros que pueden adquirir distintos valores. Estos parámetros se pueden combinar de forma aleatoria entre ellos o podemos pensar en una combinación pseudoaletoria según el valor tomado por uno de ellos. A continuación se puede ver un ejemplo de un ejercicio base con sus dos posibles redacciones y sus parámetros:

* El diagnóstico de una determinada enfermedad $M$ se realiza mediante una prueba de ultrasonidos. La sensibilidad de esta técnica es del P1\% y su especificidad del P2\%. Se sabe que esta enfermedad afecta al P3\% de una determinada población. Se escoge al azar un individuo de esta población y se le somete a la prueba de ultrasonidos. Si la prueba da P4. ¿Cuál es la probabilidad que el individuo padezca la enfermedad?

* Se ha desarrollado una nueva técnica usando ultrasonidos para la detección de una enfermedad que afecta al P3\% de una determinada población. El porcentaje de verdaderos positivos de esta técnica es del P1\% y el porcentaje de verdaderos negativos es del P2\%. Al azar se escoge un individuo de esta población y se le somete a una prueba de ultrasonidos. Si la prueba da P4. ¿Cuál es la probabilidad que el individuo padezca realmente la enfermedad?

Como podemos ver, las dos redacciones del enunciado nos piden lo mismo pero de distinto modo, y los parámetros del ejercicio podrían ser los que detallamos a continuación:

P1: 80, 85, 90, 91, 95

P2: 92, 94, 96, 98

P3: 20, 25, 30, 35, 40

P4: positivo, negativo

De este modo a partir de estas redacciones del enunciado y de estos parámetros seríamos capaces de generar 400 enunciados distintos pero que serían del mismo nivel de dificultad todos ellos.

Fijémonos que aunque cambie la redacción del enunciado o sus parámetros el método de resolución del ejercicio es el mismo, y la solución final de este vendrá determinada por los parámetros seleccionados en el enunciado. De esta forma todos los enunciados generados a partir de un ejercicio base tienen el mismo código de resolución, por lo que podemos automatizar la corrección de todos los enunciados generados.

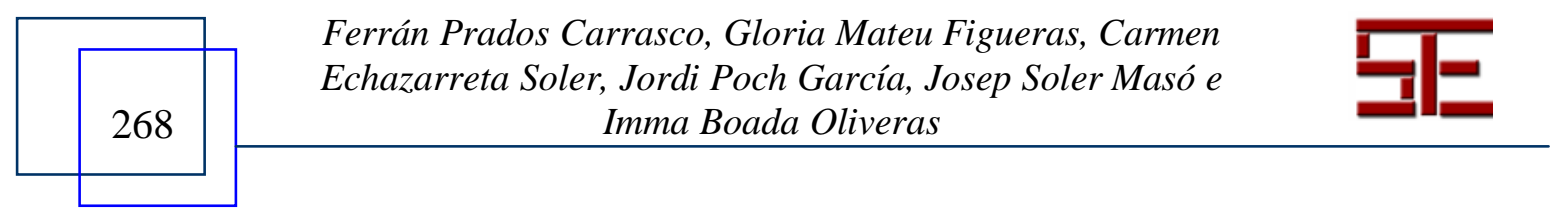




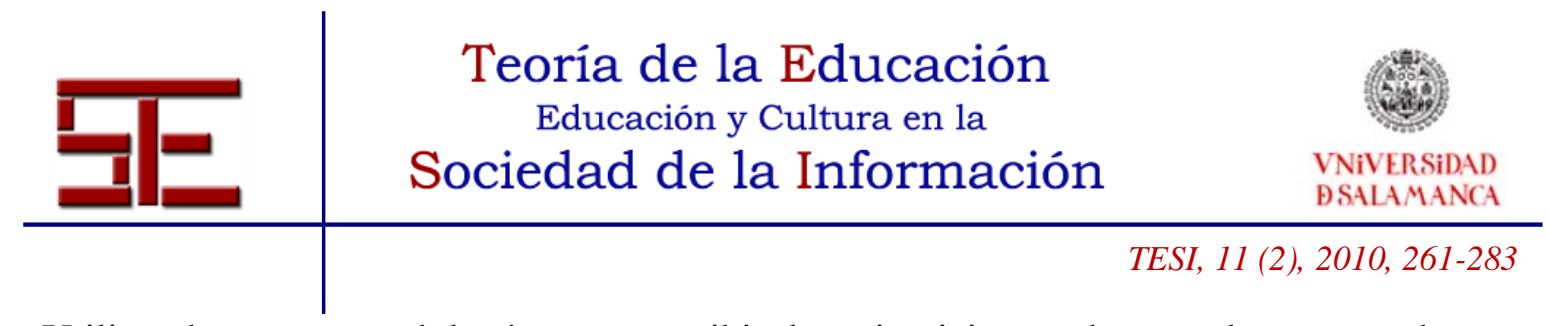

Utilizando esta metodología para escribir los ejercicios podemos obtener cuadernos personalizados de ejercicios para cada alumno. En la Figura 2 se puede observar un esquema que detalla el funcionamiento del generador de ejercicios.

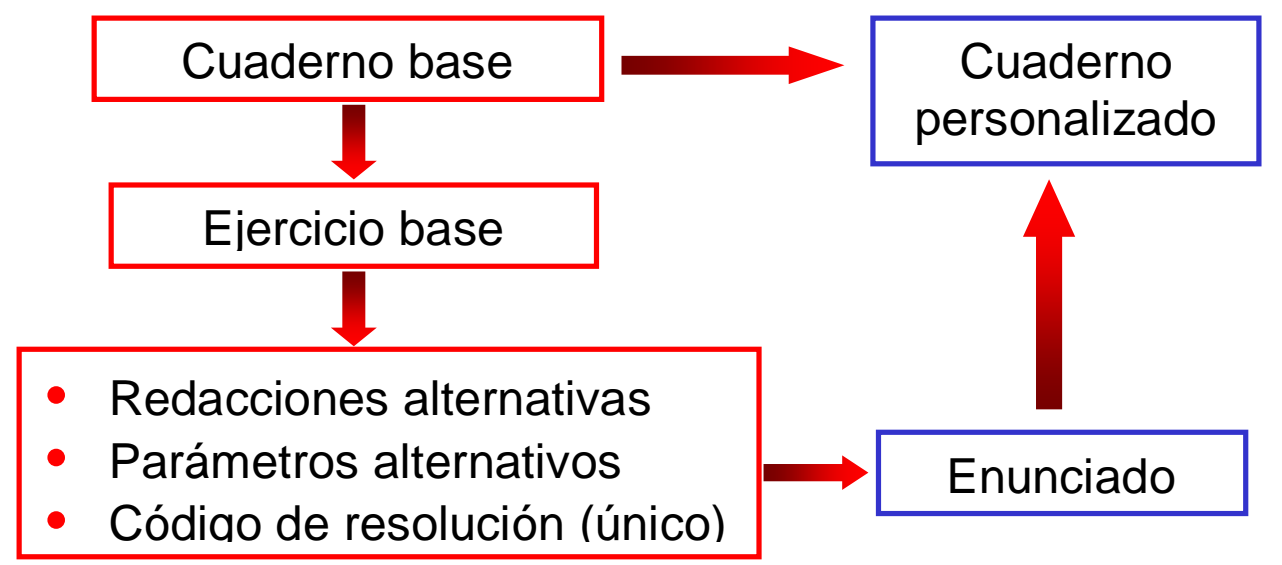

Figura 2 - Esquema del generador de ejercicios

\section{2-Formulación inorgánica}

Los ejercicios de formulación inorgánica son ejercicios, como su nombre indica, en los que se pide al alumnado que escriba la fórmula de un compuesto químico o una reacción, se caracterizan por ser muy parametrizables. Con esta tipología de ejercicios tenemos un claro ejemplo de que de un enunciado base se pueden generar miles de posibles ejercicios diferentes para los alumnos.

El alumnado debe de introducir como solución una fórmula o reacción de química inorgánica. Para facilitar la escritura de las formulas los alumnos y los profesores disponen de un editor de fórmulas muy fácil de utilizar, que no solo ayuda a la escritura de las fórmulas, sino que también tiene un carácter pedagógico ya que como se muestra en la Figura 3 dispone de una interface en forma de tabla periódica y el editor no tiene restricciones para la escritura de las fórmulas, por lo que permite al alumnado escribir fórmulas incorrectas tal y como lo podría hacer en un papel. Una vez escritas y enviadas a corregir, estas fórmulas incorrectas serán procesadas y detectadas por el corrector, y el alumno recibirá su correspondiente mensaje de feedback. Esta libertad en la escritura de las fórmulas es lo que hace aún más real y pedagógica la utilización de esta herramienta.

Ferrán Prados Carrasco, Gloria Mateu Figueras, Carmen Echazarreta Soler, Jordi Poch García, Josep Soler Masó e Imma Boada Oliveras 


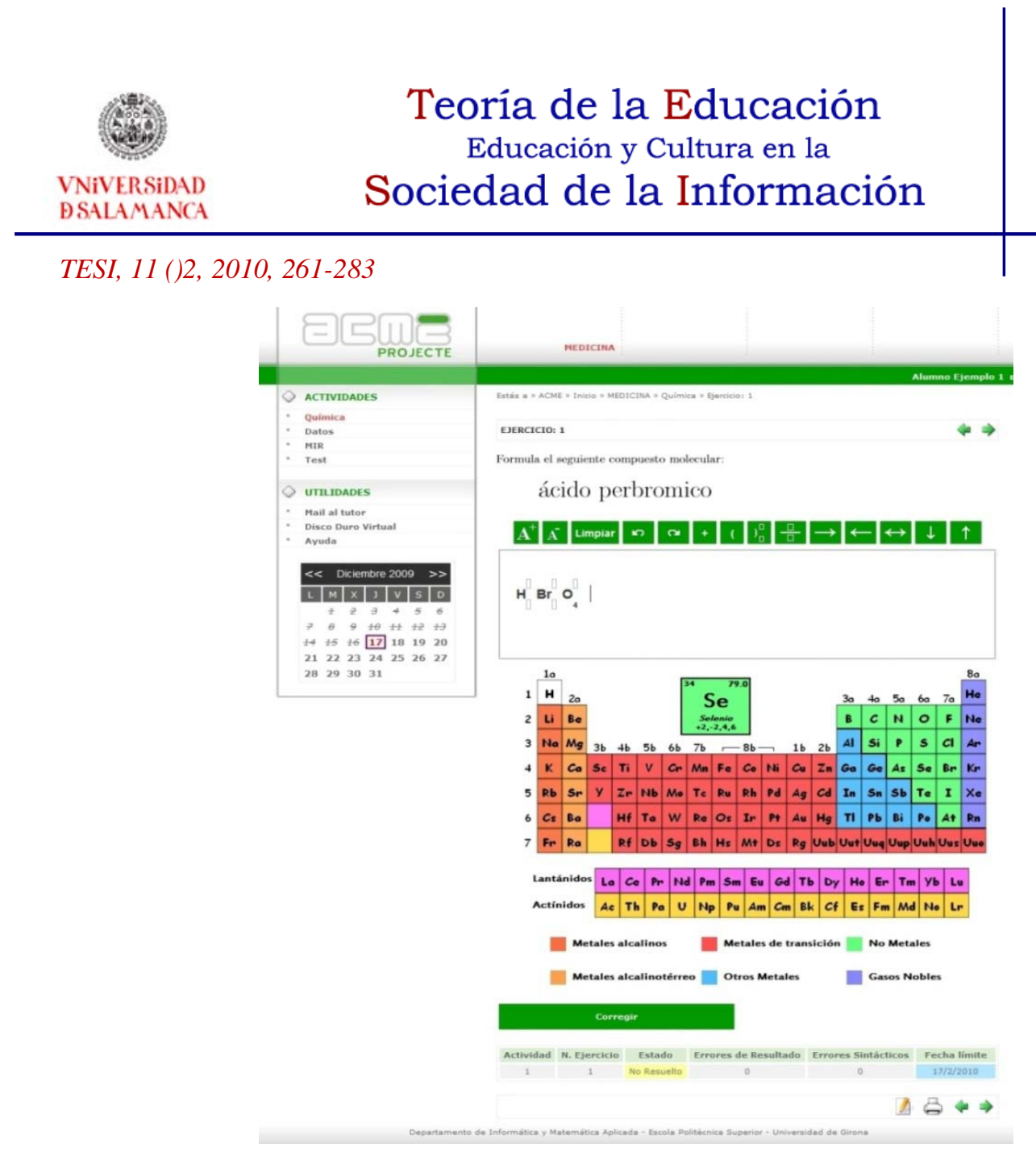

Figura 3 - Ejemplo de ejercicio de química inorgánica, se puede observar claramente la tabla periódica con la que interactúa el alumnado y la fórmula resultante que está construyendo.

\section{3-Formulación orgánica}

Esta tipología de ejercicios tiene las mismas características que la tipología de formulación inorgánica pero con una interface adaptada a formulación orgánica. Al igual que el módulo anterior los ejercicios son parametrizables y las soluciones que puede enviar el alumnado son o bien fórmulas o bien reacciones dónde intervienen moléculas de química orgánica. En este caso el editor es el popular JME desarrollado por Peter Ertl' ${ }^{2}$. Este editor de fórmulas de química orgánica se caracteriza porque las moléculas escritas por el alumnado son equilibradas, o sea, que la herramienta no permite crear cualquier molécula química sino que, según lo que dibuje el alumno el editor recalcula en tiempo real los elementos necesarios para equilibrar la fórmula y los añade. Esta pequeña ayuda resulta ser un elemento positivo a nivel pedagógico. En la Figura 4 se puede ver un ejemplo de formulación de química orgánica.

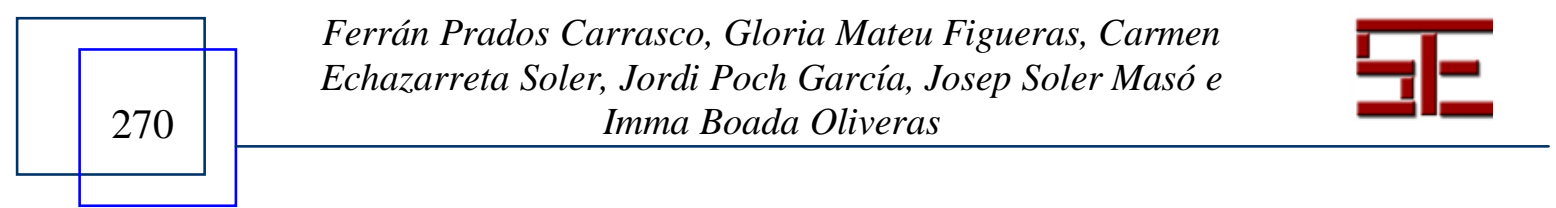




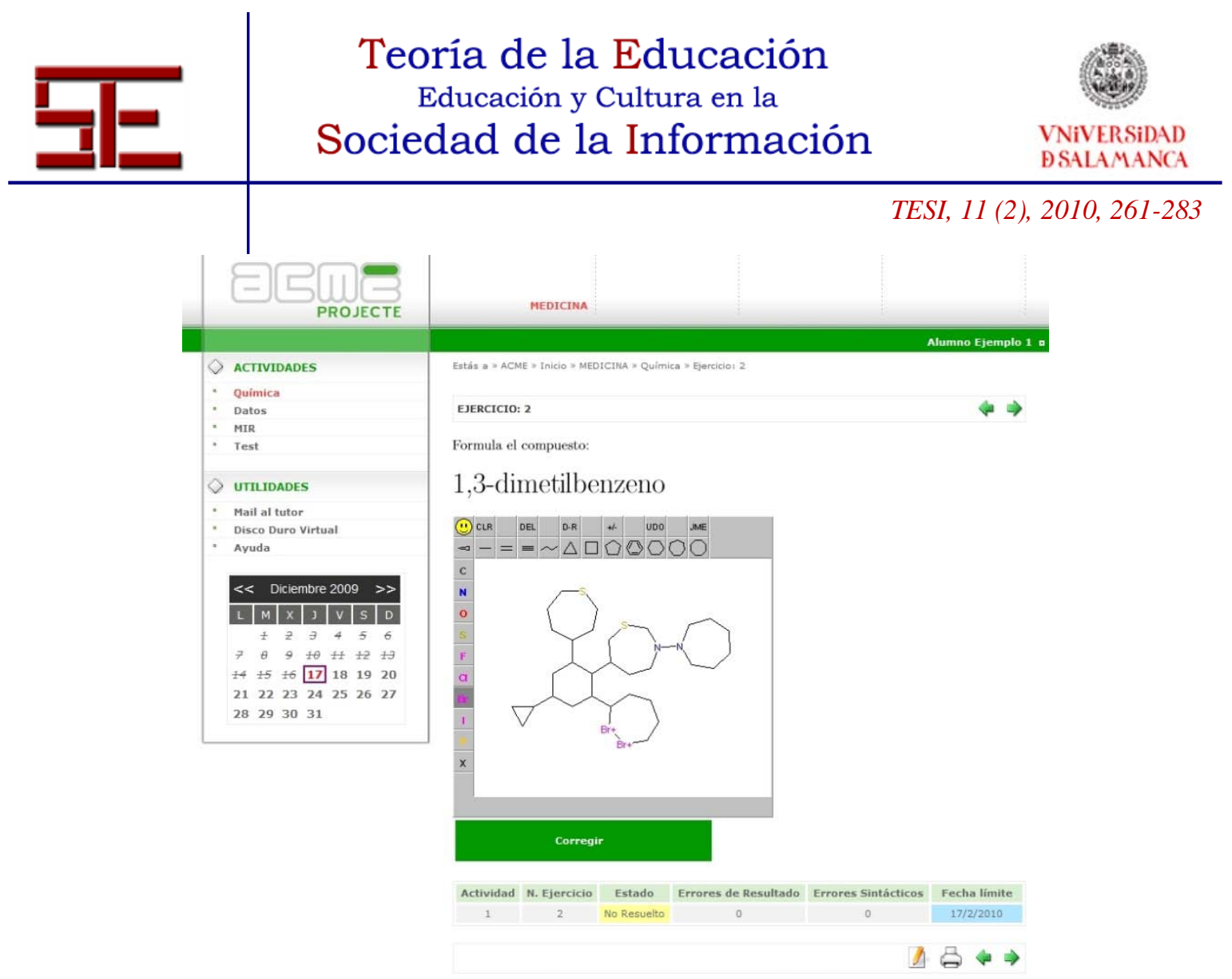

Figura 4 - Ejemplo de ejercicio de química orgánica se puede observar la aplicación con la que interactúa el alumno y la fórmula resultante que está construyendo

\section{4-Tablas de datos}

Esta tipología de ejercicio se caracteriza por el hecho que la solución es una tabla de valores y resultados con una estructura determinada, con lo que el alumno puede entrar toda una serie de datos en una tabla tipo hoja de cálculo. Esta tipología puede utilizarse en una gran variedad de ejercicios, problemas y/o prácticas. Así puede utilizarse en ejercicios de matemáticas o economía, prácticas de física, química o estadística, de hecho en un sentido amplio, puede utilizarse en cualquier caso donde sea necesario recoger unos datos experimentales y hacer un posterior análisis de estos. El módulo corrector permite filtrar los resultados experimentales verificando que se encuentren en el rango adecuado y verificar los cálculos del posterior análisis. Lo que permite al alumno verificar los resultados para su posterior interpretación y ahorra al profesor la verificación de los resultados pudiendo centrarse en la valoración de la interpretación realizada por el alumno. Estas tablas, normalmente incorporan una plantilla que ayuda al alumnado a colocar los datos en su lugar adecuado. Además, podemos proporcionar a los alumnos un fichero de datos que se puede generar con contenidos diferentes y de forma aleatoria para cada estudiante como si fuera un parámetro más del ejercicio que el

Ferrán Prados Carrasco, Gloria Mateu Figueras, Carmen Echazarreta Soler, Jordi Poch García, Josep Soler Masó e Imma Boada Oliveras 


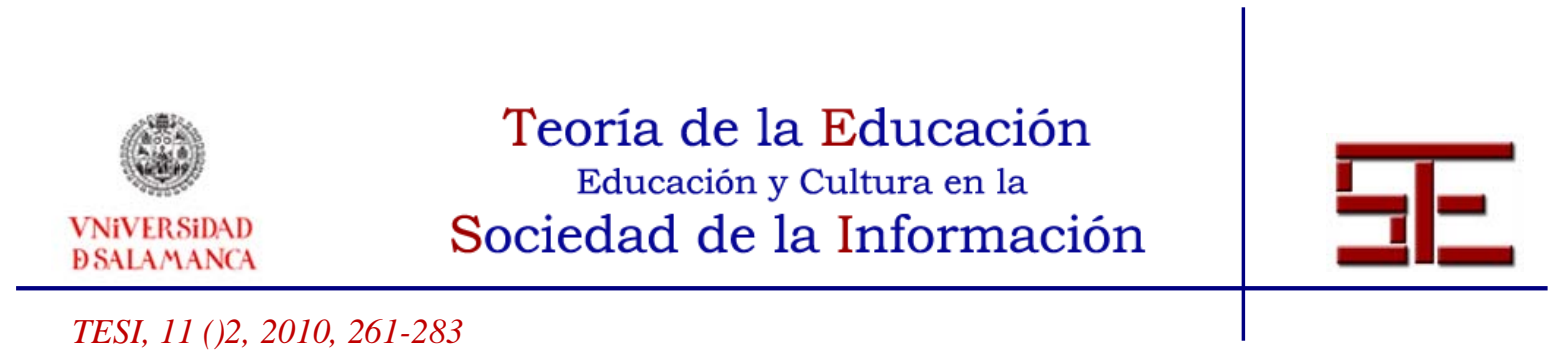

corrector tendrá en cuenta. La Figura 5 podemos ver un ejemplo de ejercicio en el que se propone que se realice un estudio estadístico.

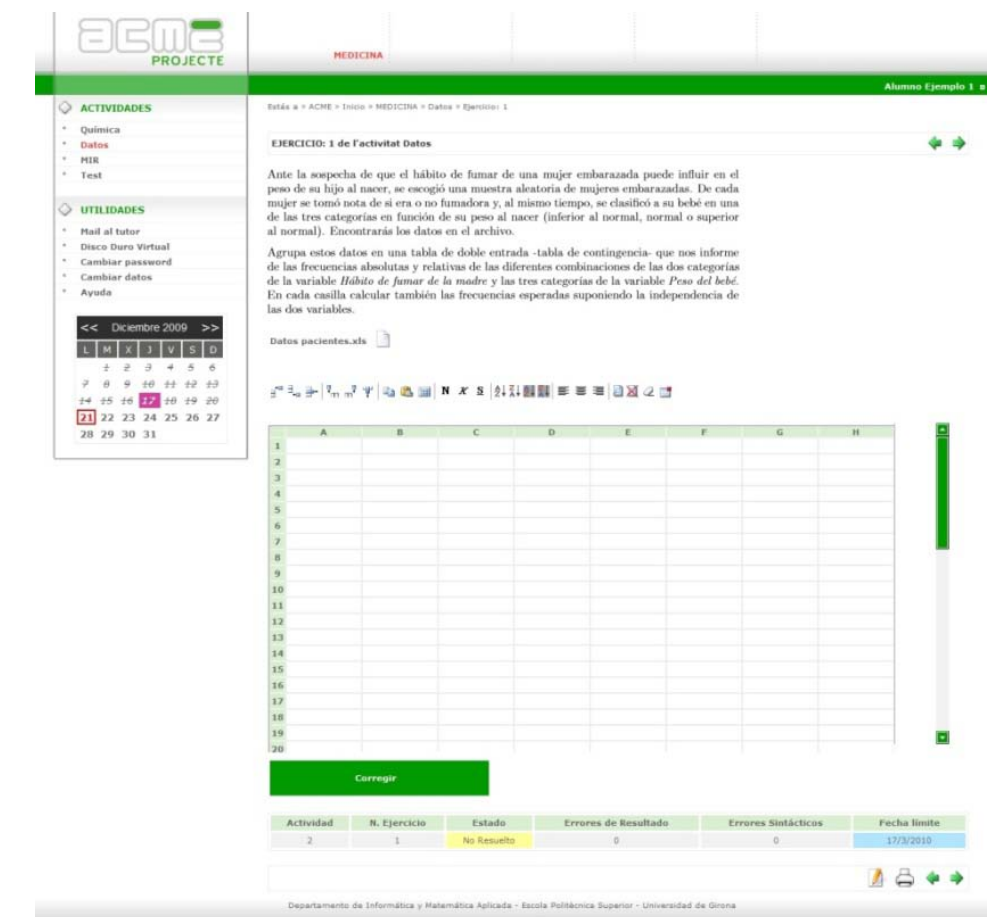

Figura 5 - Ejemplo de ejercicio donde el resultado puede ser una tabla de datos

\section{5-Verdadero - falso}

Los ejercicios de verdadero - falso consisten en una lista de afirmaciones que el alumno debe identificar como ciertas o falsas. Tienen múltiples aplicaciones en el análisis de casos o situaciones. Pueden llevar adjunto un texto o una imagen. Así por ejemplo podemos realizar una serie de afirmaciones sobre un texto o imagen y pedir al alumnado que responda si son ciertas o falsas las afirmaciones. Por lo que podemos utilizarlo en interpretación de textos o imágenes y estudio de casos para la extracción de conclusiones. En la Figura 6 se muestra un ejemplo en el que se realizan una serie de afirmaciones relacionadas con una imagen radiológica. Estos ejercicios no tienen parámetros, pero sí pueden contener numerosas afirmaciones lo que nos permite de igual modo generar ejercicios distintos para cada alumno variando el orden y la lista de afirmaciones escogidas. Una de las ventajas de este tipo de ejercicio es la simplicidad de escritura, con lo que es muy fácil de preparar por parte del profesor. En cuanto al alumnado el sistema les proporciona una nota siguiendo los criterios fijados por el profesor.

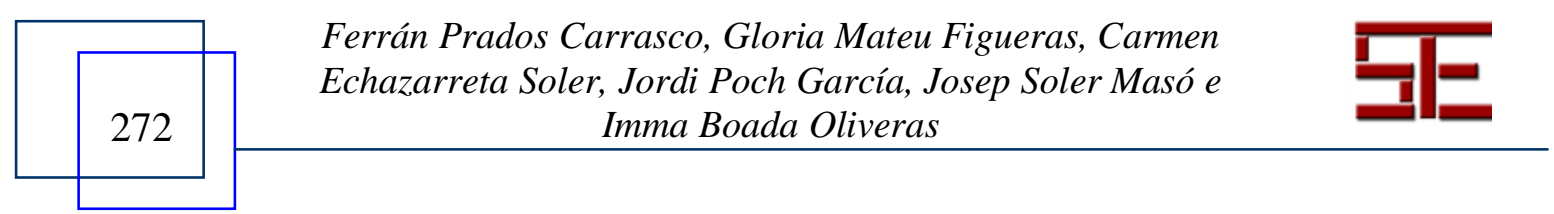




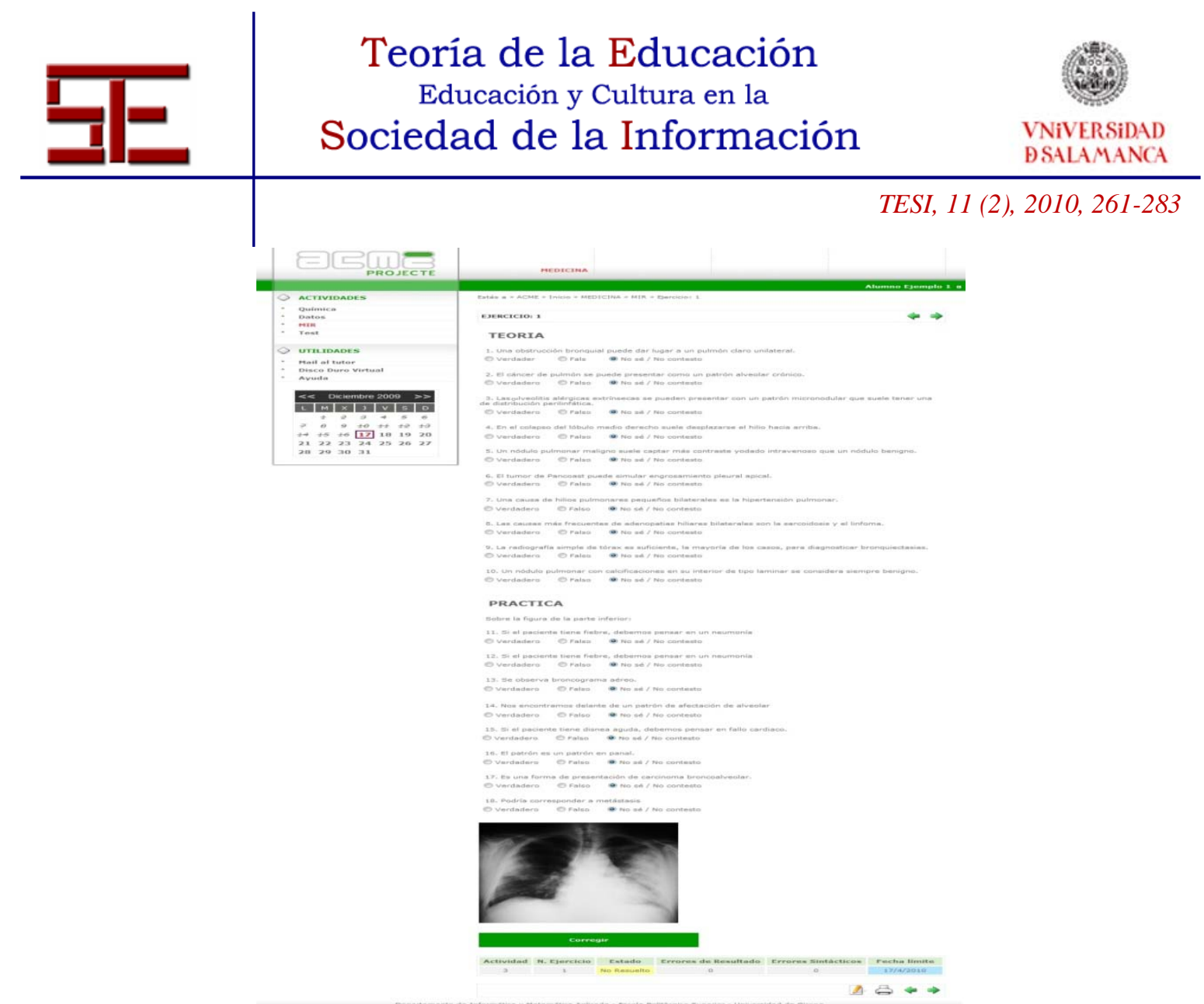

Figura 6 - Ejemplo de visualización por parte del alumno de un ejercicio de verdadero o falso

Los ejercicios de verdadero y falso se han mostrado muy útiles en el campo de las ciencias de la salud para que los alumnos demuestren sus conocimientos evaluando casos. El procedimiento que sigue es el siguiente, al alumno se le muestra por ejemplo una imagen radiológica y se le plantean toda una serie de afirmaciones o frases que debe indicar si son verdaderas o falsas, o bien no sé/no contesto. El objetivo final es que llegue a evaluar la patología del caso presentado planteando todos los hitos que tendría que mirar cuando esté frente a un caso real.

\section{6-Test}

Los ejercicios test, consistentes en seleccionar la respuesta correcta entre diversas opciones, que se pueden hacer en el ACME son los típicos ejercicios test que encontramos en otras plataformas de e-learning pero con la posibilidad de ser parametrizados. Según el valor de los parámetros puede variar la respuesta correcta y el número de respuestas correctas. El número de respuestas que se muestran puede variar y el ejercicio puede tener más respuestas alternativas que la que se muestran. Todo esto junto con el generador de enunciados le da mayor potencia puesto que permite generar

Ferrán Prados Carrasco, Gloria Mateu Figueras, Carmen Echazarreta Soler, Jordi Poch García, Josep Soler Masó e Imma Boada Oliveras 


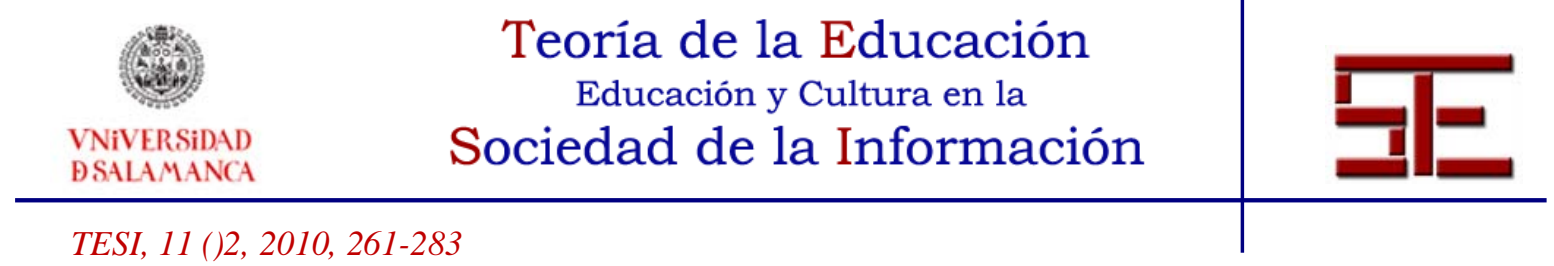

versiones distintas para cada estudiante. La Figura 7Figura 5 podemos ver un ejemplo de ejercicio en el que se propone que se realice un estudio estadístico.

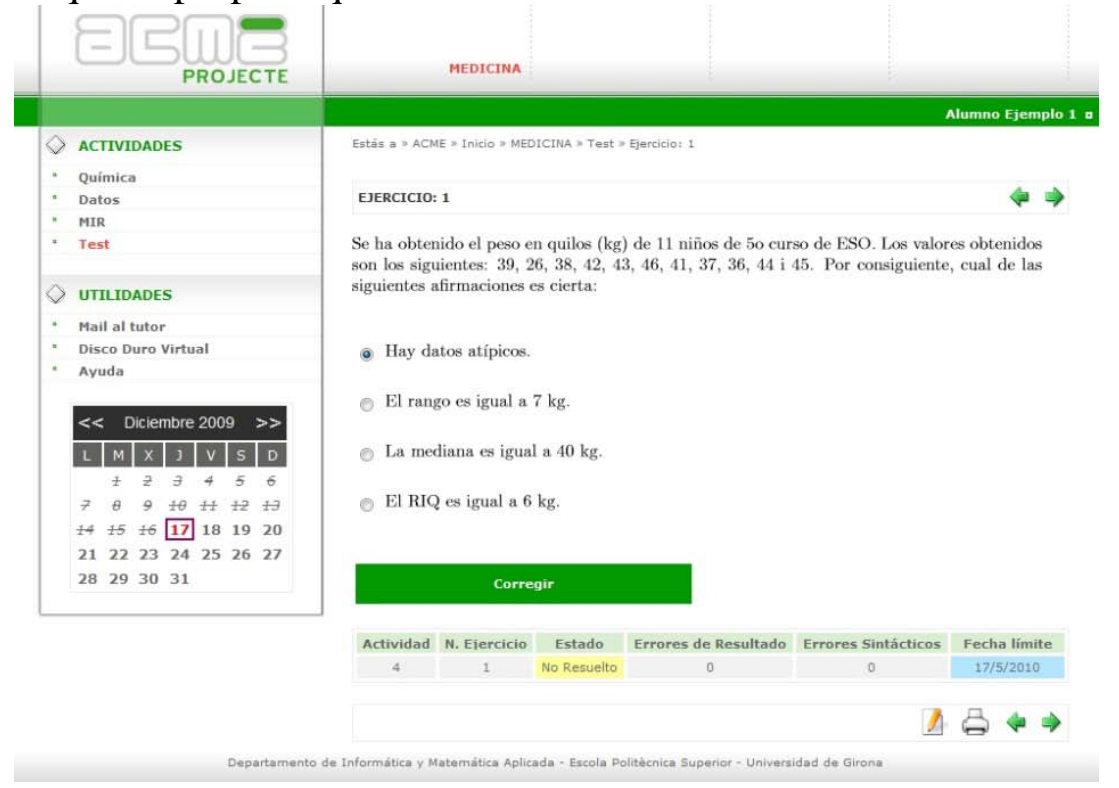

Figura 7 - Ejemplo de visualización por parte del alumno de un ejercicio tipo test

\section{3.- LAS COMPETENCIAS EN EL ÁMBITO DE LAS CIENCIAS DE LA SALUD}

El concepto competencia y su papel en la formación de los estudiantes universitarios es un elemento clave en el Espacio Europeo de Educación Superior (EEES). El proyecto Tuning $^{3}$ considera que las competencias son las capacidades y habilidades que las personas necesitan para moverse en todos los contextos que les sean propios y las sitúa en el currículum, en las aulas, y en la planificación de la docencia:

"Competences represent a dynamic combination of knowledge, understanding, skills and abilities. Fostering competences is the object of educational programmes. Competences will be formed in various course units and assessed at different stages” (Proyecto Tuning).

Asimismo, el Comunicado de Berlín ${ }^{4}$ de la conferencia de ministros de educación de la Unión Europea, pone el énfasis en establecer la compatibilidad de los títulos, uno de los objetivos de la armonización europea, en base al enunciado de las competencias que cada titulado haya adquirido:

"Los ministros animan a los estados miembros a elaborar un marco de calificaciones comparables y compatibles para sus sistemas de educación superior, que tenga como objetivo la descripción de las calificaciones en términos de carga de trabajo, nivel, resultados de

$274 \quad\left[\begin{array}{c}\text { Ferrán Prados Carrasco, Gloria Mateu Figueras, Carmen } \\ \text { Echazarreta Soler, Jordi Poch García, Josep Soler Masó e } \\ \text { Imma Boada Oliveras }\end{array}\right.$




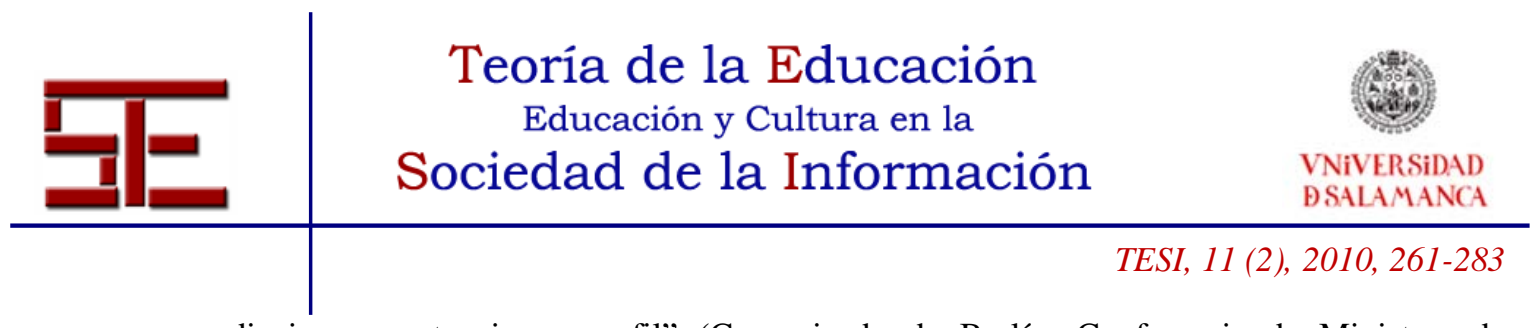

aprendizaje, competencias y perfil” (Comunicado de Berlín, Conferencia de Ministros de Educación de la Unión Europea).

En este contexto normativo, la incorporación de las competencias a los estudios universitarios resulta un elemento básico para la formación, en una sociedad cambiante que reformula sus demandas constantemente y que a su vez aspira a profesionalizar la formación universitaria acercando la universidad a la sociedad y, en nuestro caso, al campo de la salud.

Esencialmente, las competencias transversales son las relacionadas con el desarrollo personal, no dependen de un ámbito temático o disciplinario específico sino que penetran todos los dominios de la actuación profesional y académica. Son las competencias relacionadas con el saber ser y el saber estar y se refieren a las habilidades personales, sociales y/o metodológicas que en el Marco Europeo de Cualificaciones ${ }^{5}$ se describen en términos de responsabilidad y autonomía. La Agencia de la Calidad Universitaria de Cataluña ha definido las competencias transversales o genéricas que deberían conseguir todos los licenciados en medicina ${ }^{6}$. Las que proponemos a continuación son el resultado de fusionar el documento del AQU Cataluña con las competencias del proyecto Tuning:

1. Resolver problemas complejos de forma efectiva en el campo de la salud

2. Aplicar pensamiento crítico, lógico y creativo, demostrando dotes de innovación.

3. Trabajar de forma autónoma con responsabilidad e iniciativa.

4. Trabajar en equipo de forma colaborativa y responsabilidad compartida.

5. Liderar y gestionar proyectos técnico-sanitarios

6. Comunicar información, ideas, problemas y soluciones de manera clara y efectiva en público o ámbito técnico concretos.

7. Mostrar dominio del inglés en todos los ámbitos de uso de esta lengua

8. Saber utilizar con seguridad y destreza las TIC.

A continuación vamos a analizar estás competencias y como una herramienta de corrección automática nos puede ayudar en el trabajo de las mismas.

\section{Resolver problemas complejos de forma efectiva en el campo de la salud}

Esta competencia es de las consideradas de primer nivel pues se refiere a la toma de decisiones frente a la que se halla frecuentemente el profesional de la salud. Por lo tanto se trata de una habilidad que debe ser entrenada en el ámbito universitario. En este sentido, ACME, y otras herramientas de corrección automática, nos promueven entornos pertinentes para el aprendizaje y la evaluación de esta competencia. Por

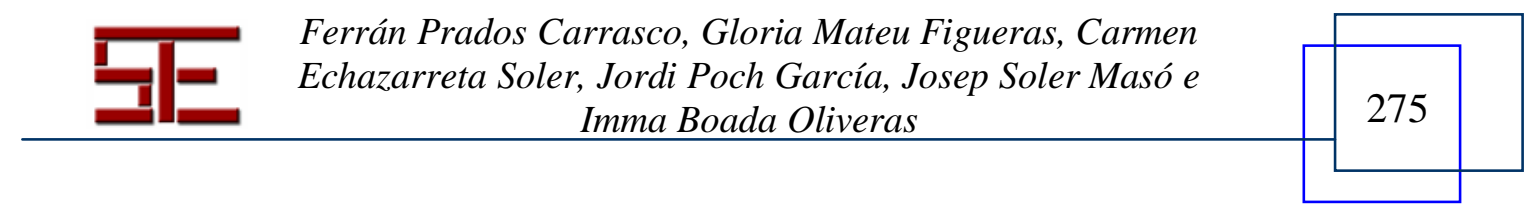




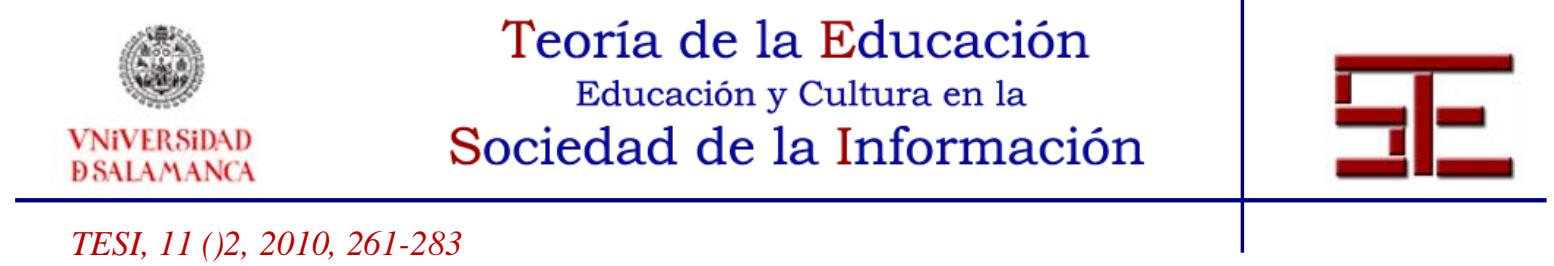

ejemplo ACME con su multitud de tipologías de ejercicios nos permite plantear actividades de una elevada complejidad dónde se deba de combinar ejercicios individuales y de trabajo en grupo, con diferentes tipos de corrección y que se puedan hacer en clase, en prácticas o en casa (Prados 2009), como se puede observar en la Figura 8. Al estudiante se le debe poner en situaciones distintas en las cuales deba optar por alternativas opuestas para poder resolver un problema.

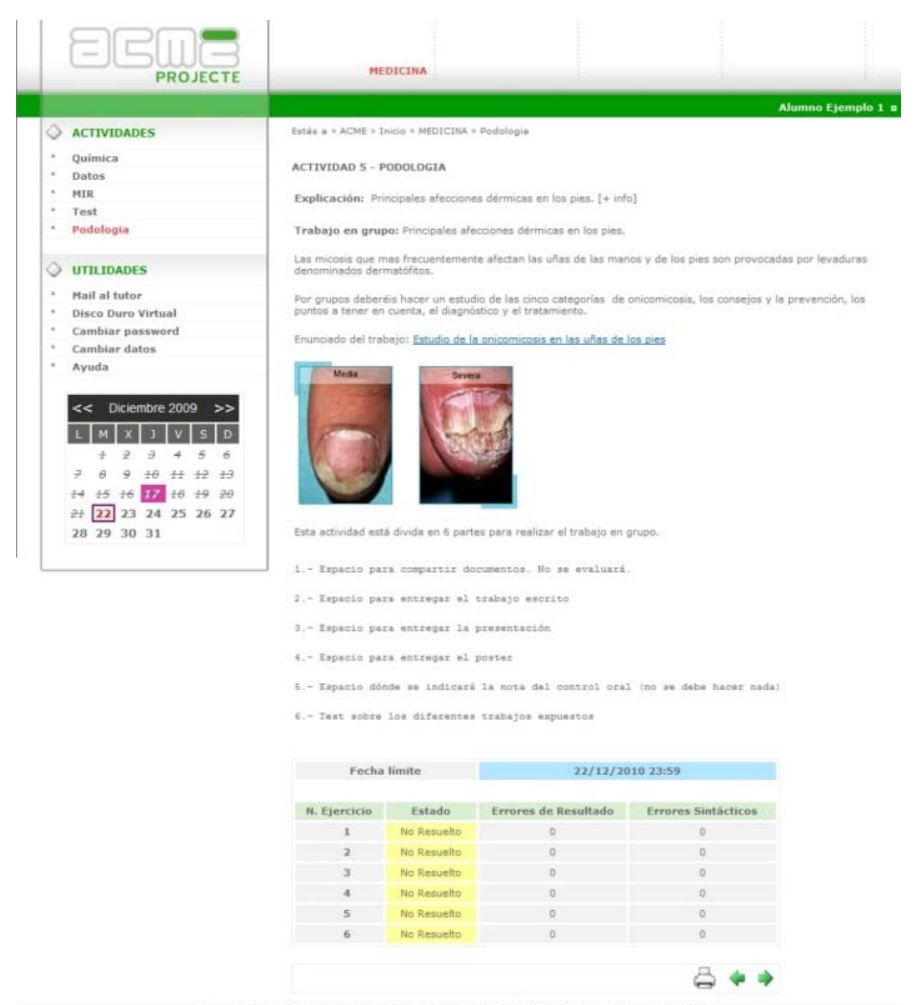

Figura 8 - Ejemplo de actividad con sus diferentes ejercicios

2. Aplicar pensamiento crítico, lógico y creativo, demostrando dotes de innovación.

Los profesionales de la salud deben desarrollar su labor en condiciones óptimas y demostrando talento y dotes de observación. En este sentido, es necesario que en su proceso de formación deban demostrar su nivel de razonamiento, de argumentación y de inquietud creativa. Sin duda, esta es una competencia necesaria pues el desempeño de la profesión reclama, además de una sólida base científica, imaginación y soluciones creativas a situaciones nuevas.

3. Trabajar de forma autónoma con responsabilidad e iniciativa.

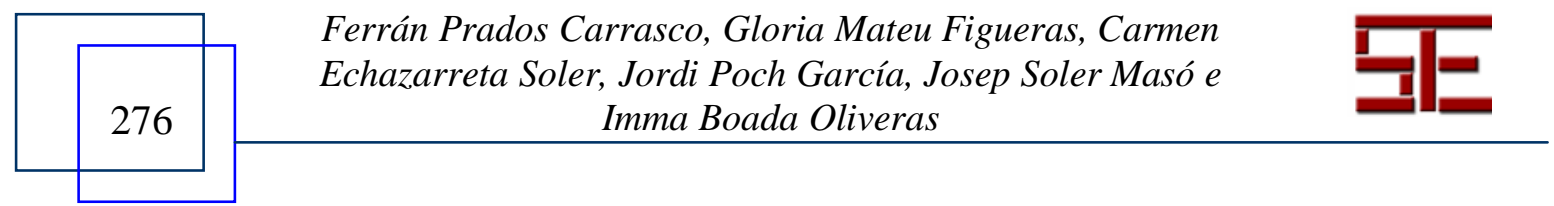




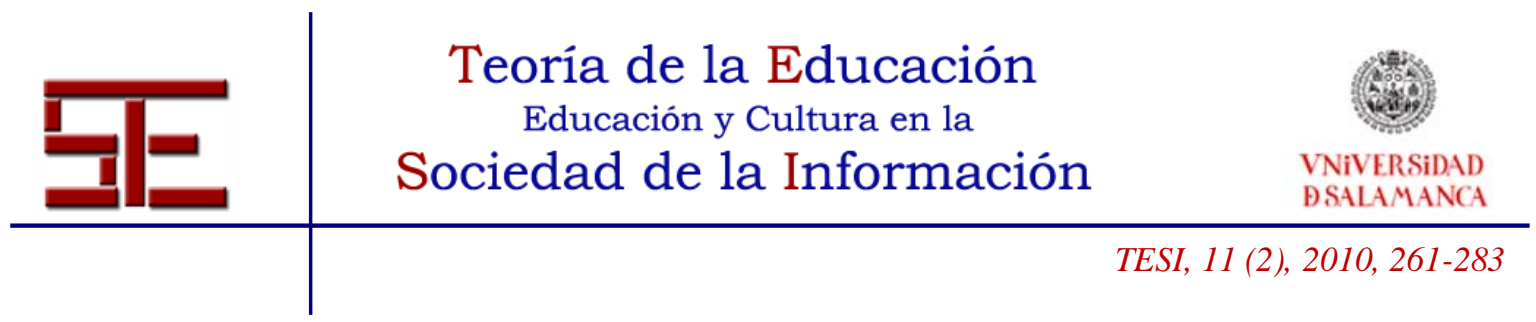

Las TIC promueven escenarios de trabajo pertinentes para el desarrollo de actividades de aprendizaje autónomo, un concepto muy próximo al autoaprendizaje. La corrección automática de ejercicios usando las TIC ayuda a fomentar el trabajo autónomo entre los alumnos.

\section{Trabajar en equipo de forma colaborativa y responsabilidad compartida.}

La globalización ha comportado la difusión de la información sin fronteras y la posibilidad de crear equipos de trabajo sin necesidad de compartir un mismo espacio. En este contexto, el desarrollo de proyectos colaborativos es una de las posibles estrategias metodológicas válidas para poner en práctica este particular enfoque de la educación, puesto que permite al alumnado aprender conjuntamente y compartir tanto responsabilidades como logros. La aplicación que presentamos en este artículo, la plataforma ACME, ha demostrado tener una gran eficacia para aumentar la motivación, mejorar el rendimiento, potenciar las habilidades sociales y desarrollar las capacidades de aprendizaje. El uso de las TIC permite superar barreras espaciales y temporales, facilitando tanto la búsqueda y el procesamiento de información como el desarrollo original de contenidos por parte de los alumnos. Asimismo, las TIC son una excelente herramienta para atender a la diversidad y para fomentar la comunicación y la expresión creativa. ACME no es solo una plataforma de corrección automática de ejercicios de forma automática sino que también permite combinar y desarrollar en una misma actividad ejercicios individuales y de trabajo en grupo como pueden ser wikis, glosarios, portafolios, blogs, webquest, etc. (Echazarreta 2009).

\section{Liderar y gestionar proyectos técnico-sanitarios}

Mostrar capacidad de gestionar las habilidades individuales para poder formar un grupo armónico, equilibrado y motivado, fomentando la lealtad y confianza entre sus miembros ${ }^{7}$.

6. Comunicar ideas, problemas y soluciones de manera clara y efectiva en ámbitos diversos.

Mostrar habilidad para exponer, de forma eficaz y correcta, los contenidos que se quieren transmitir, ya sea de forma oral o escrita, utilizando la lengua considerada como propia $^{8}$.

7. Mostrar dominio del inglés en todos los ámbitos de uso de esta lengua

La mayoría del conocimiento en ciencias de la salud se genera en lenguas ajenas a las propias y, por ello, el acceso rápido a él sólo es posible con un grado mínimo de manejo del inglés. Las grandes revistas de ciencias de la salud, las bases de datos

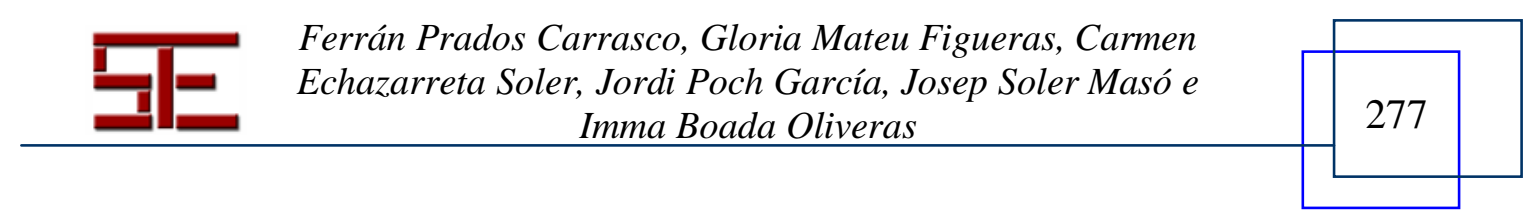




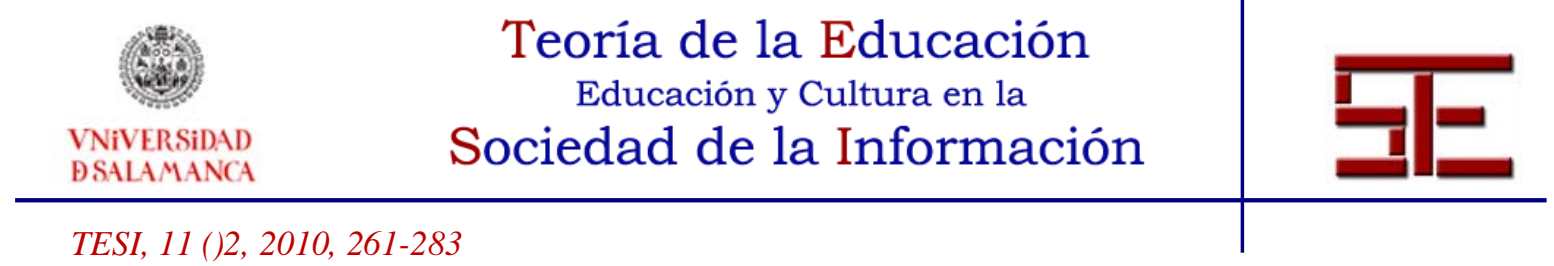

internacionales o las reuniones científicas importantes, emplean siempre esta lengua y, por ello, es indispensable para una rápida actualización de conocimientos.

8. Saber utilizar con seguridad y destreza las TIC aplicadas a diversos ámbitos del desempeño de la profesión en el campo de la salud.

Uno de los objetivos capitales de la universidad en la actual sociedad del conocimiento es la formación de profesionales competentes que dispongan de herramientas suficientes para hacer frente a las incógnitas que, paradójicamente, están asociadas a la información y a la comunicación. Y a su vez, han de ser capaces de definir y crear sus propias herramientas de trabajo con las cuales dar sentido y eficacia a este conocimiento en permanente revisión y ampliación (Echazarreta 2009).

\section{4. -CASO PRÁCTICO}

El origen de esta experiencia surgió del proceso continuo de mejora de la docencia, en el marco del EEES y de las inquietudes del profesorado para mejorar las clases y los resultados de la asignatura de bioestadística de la antigua diplomatura de enfermería de la Escola Universitària d'Infermería (EUI) de la Universidad de Girona (UdG).

El punto de partida es la asignatura de Bioestadística y metodología aplicada a la enfermería, asignatura troncal en el primer cuatrimestre del primer año con un total de 6 créditos. Actualmente, en los nuevos estudios de grado que han empezado este curso 2009/2010, los contenidos de bioestadística se han integrado en un módulo anual de primer curso denominado Bioestadística, demografía y epidemiología con un total de 9 créditos ECTS.

En las dos asignaturas mencionadas hay programadas diversas actividades con profesor, como las clases de teoría y problemas, las clases prácticas en el aula informática con grupos reducidos de 20 alumnos, las tutorías presenciales o telemáticas y en el caso de los nuevos estudios de grado, las sesiones de Aprendizaje Basado en Problemas (ABP). Referente a las actividades sin profesor hay programada la resolución de ejercicios utilizando la plataforma ACME que describiremos con detalle a continuación, la elaboración de un trabajo global de toda la asignatura con cuestiones de cierta complejidad referidas a un conjunto de datos reales y, en el caso de los estudios de grado, hay además el trabajo de autoaprendizaje de los contenidos que aparecen en problemas planteados en las sesiones de ABP.

Cuando la EUI y la UdG plantearon iniciar un plan piloto de adaptación al EEES, el profesorado de bioestadística participó activamente en la definición de una nueva estructura, definiendo las competencias y replanteando las actividades. Uno de los

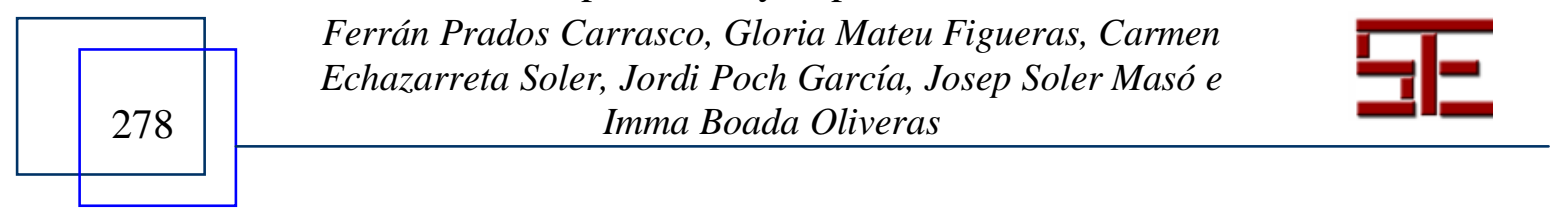




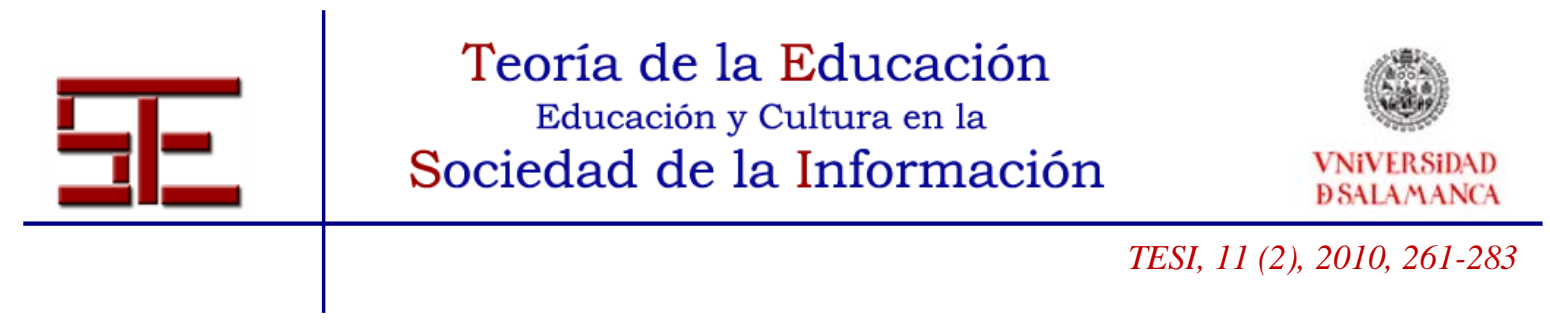

aspectos en el cual se decidió incidir fue en el control y el incentivo del trabajo continuado de los alumnos con el objetivo de incrementar su rendimiento académico final. Así, desde el curso 2006/2007 y dentro de la asignatura de Bioestadística y metodología aplicada a enfermería, se incorporaron unas actividades de aprendizaje continuado a través de la plataforma ACME. Fue necesaria una revisión exhaustiva de una gran base de problemas tipo test elaborados por los profesores del área de Estadística e Investigación operativa y una adaptación a la tipología test existente en ACME, clasificándolos por temas y por nivel de dificultad. En este proceso de adaptación de los problemas al tipo test al ACME se aprovechó para añadir una descripción y un comentario para cada problema, invisible a los alumnos, que permiten al profesor ver que objetivos tiene ese problema en concreto. Seguidamente se pasó a la implementación en el grupo de alumnos matriculados en la asignatura. El resultado fue un dossier personalizado para cada alumno. Para los alumnos de enfermería se programaron los siguientes cuatro bloques de preguntas:

1. Estadística descriptiva

2. Probabilidad y modelos

3. Inferencia estadística

4. Interpretación de un texto científico. En este bloque primero se proporcionaba al alumno un artículo científico publicado en revistas del ámbito sanitario que debía leer y entender antes de responder a las preguntas.

Cada bloque tenía unas fechas de activación y entrega prefijadas al inicio de curso que permitían a los alumnos planificar y trabajar según sus disponibilidades y conveniencias. Estos cuatro bloques tenían un peso global del 20\% de la nota de la asignatura aunque el día del examen presencial, el alumno debía validar la nota contestando a un cuestionario tipo test con la misma tipología de preguntas.

La participación de los alumnos en esta actividad fue muy satisfactoria y ha ido en aumento. Se ha pasado de un 75.5\% de participación en el curso 2006/2007 hasta un 94.1\% en el curso 2008/2009. En cuanto a los resultados académicos, creemos que el uso del sistema ACME combinado con otras mejoras planteadas en el marco del EEES ha contribuido a aumentar significativamente el rendimiento de los alumnos. Se ha pasado de un 23.9\% de aprobados en el curso 2003/2004 (curso en el que aún no se había planteado ninguna mejora en el marco del EEES) a un 53\% de aprobados en el curso 2007/2008 llegando a un 63.7\% de aprobados en el curso 2008/2009. Por otra parte hemos conseguido disminuir el número de alumnos no presentados, pasando de un $31.2 \%$ en el curso $2003 / 2004$, a un $21.4 \%$ en el curso $2007 / 2008$ y a un $15.7 \%$ en el curso 2008/2009.

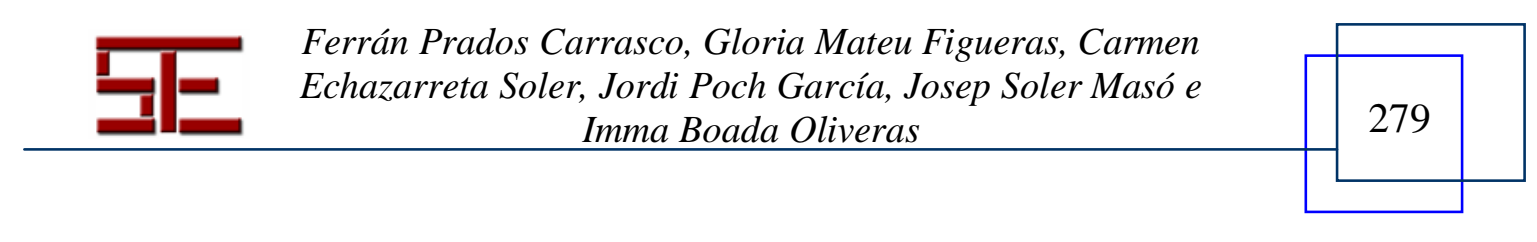




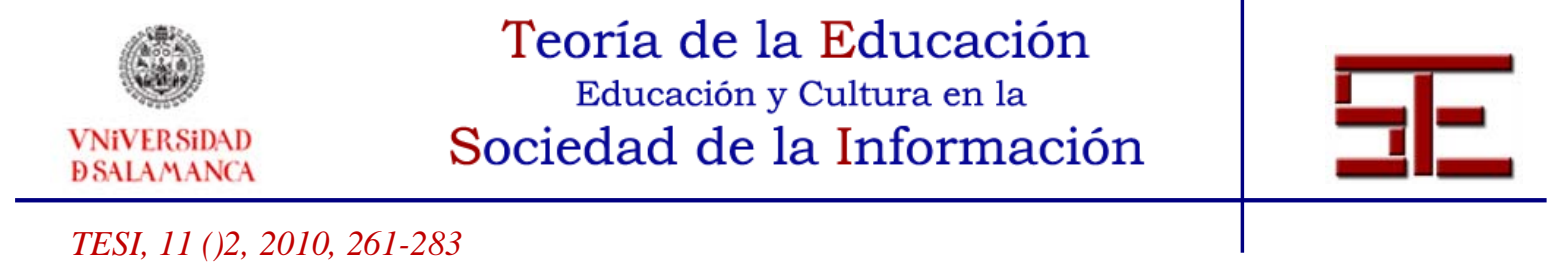

Una herramienta de seguimiento de los alumnos que proporciona el ACME son los resúmenes estadísticos por temas y por preguntas. Estos resúmenes nos han permitido hacer un seguimiento de aquellos temas donde hemos percibido más dificultades de resolución e incidir en la reformulación y presentación de éstos en clase de teoría.

Este curso 2009/2010 se imparte el primer año de los nuevos estudios de grado en enfermería. La materia de bioestadística se ha integrado en un módulo anual y más amplio. Debido a los buenos resultados obtenidos con el sistema ACME se ha decidido continuar con su utilización aunque se han planteado algunos cambios. En este curso se han preparado 3 bloques de ACME:

1. Estadística descriptiva

2. Ley normal e inferencia estadística

3. Interpretación de un texto científico.

Estos 3 bloques tienen un peso del 15\% en la nota global del módulo y el alumno también debe validar la nota el día del examen presencial. Una novedad introducida en este curso es la realización de un examen presencial en el aula informática a través de la plataforma ACME. En este caso, todos los alumnos responderán a las mismas preguntas. También se ha ampliado la tipología de las preguntas puesto que se han preparado bloques con preguntas tipo test y preguntas de verdadero/falso. Actualmente estamos trabajando en la elaboración de preguntas para el tercer bloque de manera que se puedan integrar los contenidos de las partes de demografía y epidemiología y los alumnos puedan interpretar un texto científico en su totalidad. Para el próximo curso nos hemos planteado crear bloques específicos para los contenidos de demografía y epidemiología. En estos momentos no tenemos aún resultados académicos, solamente disponemos del porcentaje de alumnos que ha decidido participar en el primer bloque, que ha sido del $95.71 \%$ y que valoramos muy positivamente.

\section{5.-CONCLUSIONES}

La corrección automática de ejercicios es una muy buena herramienta para mejorar el día a día del aprendizaje del alumno. La posibilidad de poder generar enunciados personalizados y corregirlos de forma instantánea ayuda a aumentar el rendimiento de los alumnos y a focalizar mejor el trabajo de seguimiento del profesor.

La utilidad de la corrección automática se ve aumentada si se aplica en grupos grandes, ya que abre a los profesores la oportunidad de hacer un seguimiento exhaustivo de todo el grupo, sin que le deba suponer una dedicación extra, ya que de la parte de corrección

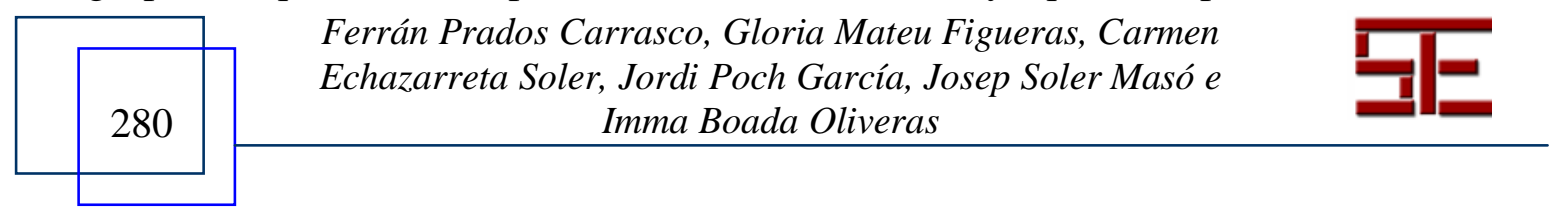




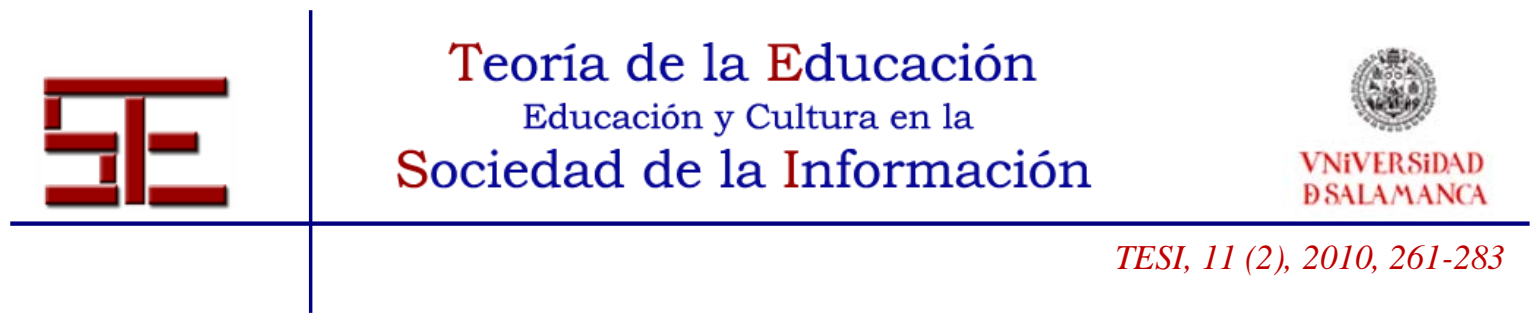

de cada ejercicio se encarga la propia herramienta. El profesor por su parte lleva a cabo una tarea más de seguimiento y revisión.

Estás herramientas nos sirven para trabajar de forma eficaz gran parte de las competencias transversales de los estudios universitarios, y en particular los del ámbito de las ciencias de la salud. Aspectos como el trabajo autónomo, planteamiento complejos de problemas, etc. se pueden trabajar utilizando correctores automáticos de ejercicios, a su vez también nos brindan la posibilidad de que los alumnos se desarrollen con soltura y seguridad en la utilización de las TIC.

La experiencia con la plataforma ACME en los estudios de Enfermería ha resultado altamente satisfactoria y ha dado unos resultados académicos realmente buenos pasando del 23.9\% de aprobados en el curso 2003/04 al 63.7\% en el 2008/09. También valoramos muy positivamente la acogida que ha tenido entre profesores del ámbito que no conocían su funcionamiento.

\section{6.-PERSPECTIVAS DE FUTURO}

La utilización de la corrección entre iguales puede ser una herramienta más a incorporar como complemento a la corrección automática. Con la corrección entre iguales (alumno-alumno) podemos trabajar algunas competencias que la corrección automática no nos permite. La corrección por iguales se está utilizando como una metodología efectiva de trabajo de ejercicios con valoraciones subjetivas.

Más concretamente en el ámbito de la ciencias de la salud. El avance de las herramientas educativas, específicas de este ámbito, no pueden quedarse al margen de Internet. Es por eso que el desarrollo de visores tridimensionales anatómicos que funcionen vía web es un punto que se debe tener en cuenta para la propuesta de nuevos correctores automáticos. Por ejemplo podríamos pensar en herramientas que permitan seleccionar un tejido en un modelo tridimensional y decirte si la selección es correcta o incorrecta. O bien simuladores virtuales tridimensionales de operaciones, etc.

A su vez tampoco podemos olvidarnos de la incorporación de laboratorios virtuales pensados para evaluar, así como otros tipos de ejercicios más técnicos, que permitirá a al profesorado del ámbito de las ciencias de la salud incorporar a los programas de sus asignaturas herramientas de corrección automática, y como resultado les simplificará el seguimiento diario de su alumnado.

En un último nivel podemos pensar en el uso de la inteligencia artificial para desarrollar correctores automáticos progresivos que aprendan a medida que los alumnos vayan

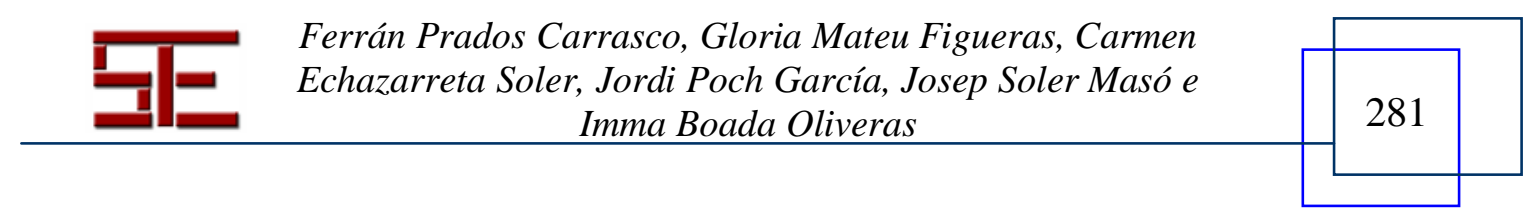




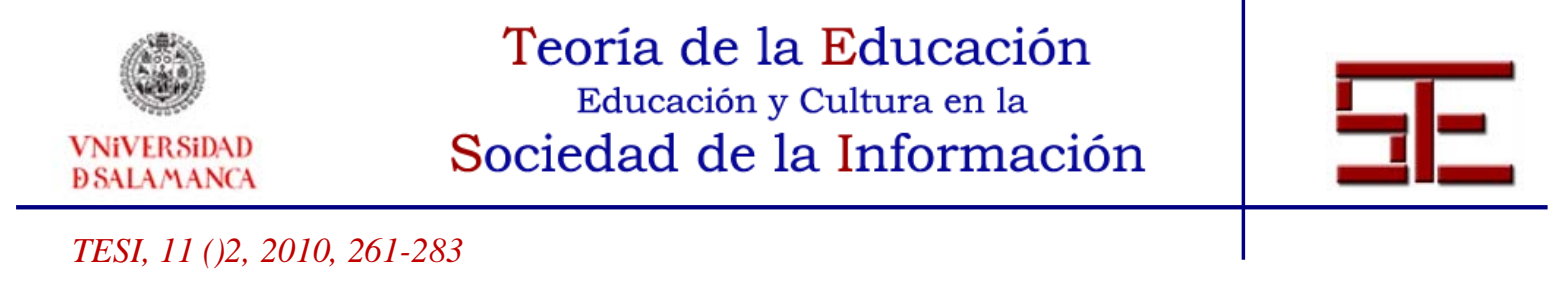

aportando soluciones y los profesores vayan corrigiendo las soluciones ambiguas. Estos correctores se basan en que si saben corregir la respuesta la corrigen y le dicen al alumno si la respuesta era correcta o incorrecta, y si no la saben corregir piden la opinión del profesor experto. A partir de la opinión dada por el profesor sobre la respuesta que no sabe el corrector, este "aprende" y de este modo amplia su conocimiento para corregir el ejercicio. Con el tiempo, y a medida que va añadiendo soluciones a su árbol de decisión, este corrector se va convirtiendo en un corrector automático.

\section{7.-BIBLIOGRAFÍA O REFERENCIAS}

Barberá, E., Bautista, G., Espasa, A. Y Guash, T. (2006). Portfolio electrónico: desarrollo de competencias profesionales en la red. RUSC Revista de Universidad y Sociedad del Conocimiento, 3(2). UOC.

Benito, A. Y Cruz, A. (2005). Nuevas claves para la docencia universitaria. Madrid: Narcea.

Boada, I., Soler, J., Prados, F. Y Poch, J. (2004). A teaching/learning support tool for introductory programming courses, ITHET 2004, Istanbul, 604-609.

Bruns, A. \& Humphreys, S. (2005). Wikis in Teaching and Assessment: The M/Cyclopedia Project, International Symposium on Wikis, 25-32.

Buzzell, P., Chamberlain, V. Y Pintauro, S. (2002). The effectiveness of web-based, multimedia tutorials for teaching methods of human body composition analysis, Advances in Physiology Education, 26, 21-29.

Davidovitch, L., Parush, A. \& Shtub, A. (2006). Simulation-based Learning in Engineering Education: Performance and Transfer in Learning Project Management, Journal of Engineering Education.

Echazarreta, C., Prados, F., Poch, J. Y Soler, J. (2009). La competencia "El trabajo colaborativo": una oportunidad para incorporar las TIC en la didáctica universitaria. Descripción de la experiencia con la plataforma ACME (UdG). UOC Papers. Número 8.

Esteve, F. (2009). Bolonia y las TIC: de la docencia 1.0 al aprendizaje 2.0. La Cuestión Universitaria, 5, 59-68.

Lammers, R. Et AL. (2008). Teaching and Assessing Procedural Skills Using Simulation: Metrics and Methodology, Academic Emergency Medicine, 15(11) 1079-1087.

Paz, M. (2008): Plataformas de Campus Virtual con herramientas de software libre: Análisis comparativo de la situación actual en las universidades Españolas. Murcia, MEC.

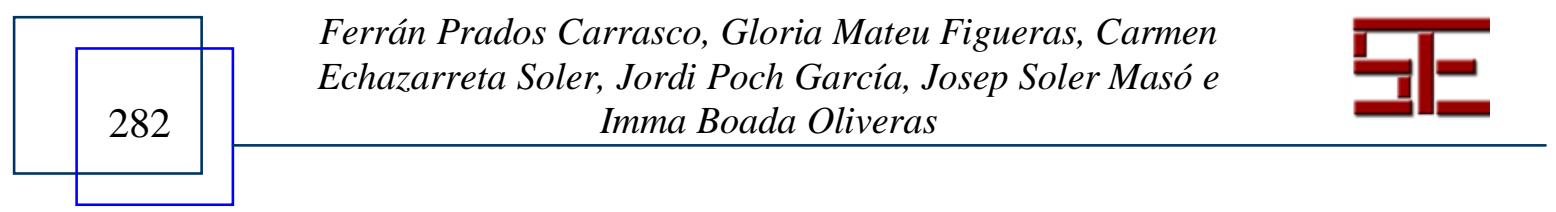




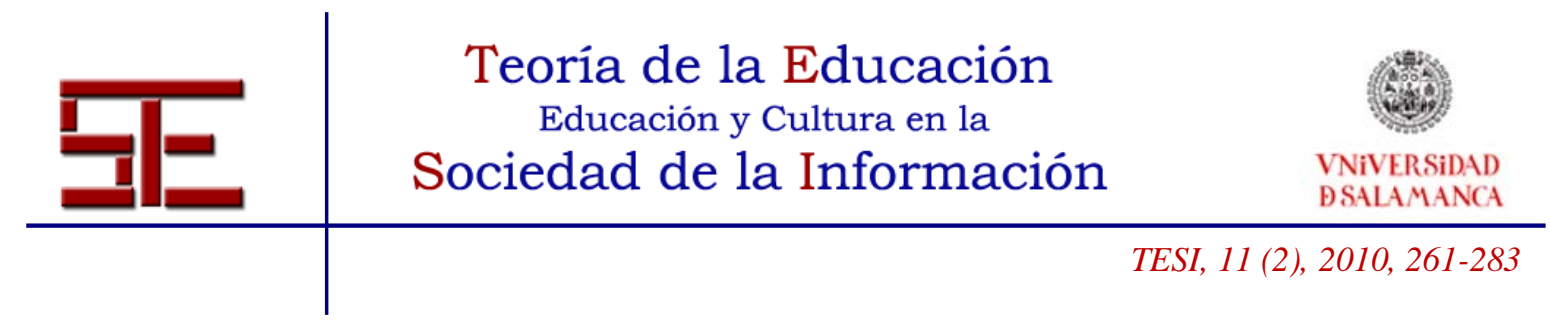

Prados, F., Boada, I., Soler Masó, J. Y Poch, J. (2005). Automatic generation and correction of technical exercises. ICECE 2005, Madrid.

Prados, F., Boada, I., Soler Masó, J., Soler Villanueva, J. Y Poch, J. (2004). El elearning como complemento a las clases presenciales un caso práctico: el proyecto ACME. Virtual Educa 2004, Barcelona.

Prados, F., Echazarreta, C., Boada, I., Soler, J. Y Poch, J. (2009). Mixing Individual and Group Activities in the same Web-Based Platform, m-ICTE2009, Lisboa.

Prados, F., Poch, J., Echazarreta, C., Boada, I. Y Soler, J. (2008). Experiències amb glossaris, wikis i portafolis amb el suport de la plataforma ACME. CIDUI 2008, Lleida.

Soler, J., Boada, I., Prados, F., Poch, J. Y Fabregat, J. (2009). Database design using a web-based e-learning tool, MCCE 2009, Barcelona.

Soler, J., Prados, F., Boada, I. Y Poch, J. (2006): Utilización de una plataforma de elearning en la docencia de bases de datos, XII Jornadas de Enseñanza Universitaria de la Informática (JENUI 2006), Bilbao.

Soler, J., Poch, J., Barrabes, E., Juher, D. Y Ripoll, J. (2002). A tool for the continuous assessment and improvement of the student's skills in a mathematics course, TICE 2002, 105-110.

Spence, L., Ferguson, R., Parrington, N. Y Thompson, J. (1994). The use of hypertext and the world-wide-web in teaching software engineering, Active Learning.

\section{Notas}

1- http://calculadora.edu365.cat

2- http://www2.chemie.uni-erlangen.de/services/fragment/editor/index.html

3-http://tuning.unideusto.org/tuningeu/index.php?option=com_frontpage\&Itemid=1

4- http://www.udg.edu/Portals/49/Docencia\%202010/Berlin_communique_190903.pdf

5-http://www.europarl.europa.eu/sides/getDoc.do?pubRef=-//EP//TEXT+TA+P6-TA-2007-

0463+0+DOC+XML+V0//ES\#BKMD-21

6-http://www.aqu.cat/actualitat/noticies/n2349.html

7-http://www.aqu.cat/doc/doc_21160857_1.pdf

8-http://www.aqu.cat/doc/doc_21160857_1.pdf

Para citar el presente artículo puede utilizar la siguiente referencia:

Prados Carrasco, F., Mateu Figueras, G., Echazarreta Soler, C., Poch García, J., Soler Masó, J y Boada Oliveras, I. (2010). Utilización de software de corrección automática en el campo de las Ciencias de la Salud, en Juanes Méndez, J. A. (Coord.) Avances tecnológicos digitales en metodologías de innovación docente en el campo de las Ciencias de la Salud en España. Revista Teoría de la Educación: Educación y Cultura en la Sociedad de la Información. Vol. 11, $\mathrm{n}^{\circ}$ 2. Universidad de Salamanca, pp. 261-283 [Fecha de consulta: dd/mm/aaaa]. http://campus.usal.es/ revistas_trabajo/index.php/revistatesi/article/view/7080/7113

Ferrán Prados Carrasco, Gloria Mateu Figueras, Carmen Echazarreta Soler, Jordi Poch García, Josep Soler Masó e Imma Boada Oliveras 


\begin{tabular}{lll} 
Teoría de la Educación & $\begin{array}{c}\text { Tducación y Cultura en la } \\
\text { VNiVERSIDAD } \\
\text { DSALAMANCA }\end{array}$ \\
\hline TESI, 11 ()2, 2010, 261-283 & Sociedad de la Información
\end{tabular}

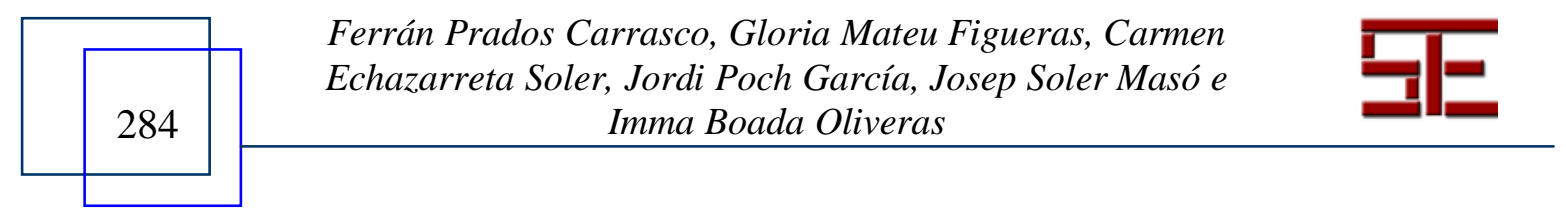

